\title{
Enantioselective Synthesis of Axially Chiral Biaryls through Rhodium-Catalyzed Complete Intermolecular Cross Cyclotrimerization of Internal Alkynes
}

\author{
Ken Tanaka, ${ }^{* \dagger}$ Goushi Nishida, ${ }^{\dagger}$ Masakazu Ogino, ${ }^{\dagger}$ Masao Hirano, ${ }^{\dagger}$ and Keiichi \\ Noguchi \\ ${ }^{\dagger}$ Department of Applied Chemistry, Graduate School of Engineering, Tokyo University \\ of Agriculture and Technology, Koganei, Tokyo 184-8588, Japan \\ Instrumentation Analysis Center, Tokyo University of Agriculture and Technology, \\ Koganei, Tokyo 184-8588, Japan
}

\section{General}

Anhydrous $\mathrm{CH}_{2} \mathrm{Cl}_{2}$ (No. 27,099-7) was obtained from Aldrich and used as received. H8BINAP and Segphos were obtained from Takasago International Corp. All other reagents were obtained from commercial sources and used as received. All reactions were carried out under an atmosphere of argon or nitrogen in oven-dried glassware with magnetic stirring.

\section{Cross Cyclotrimerization of Diethyl Acetylenedicarboxylate and Internal Alkynes (Table 1)}

General Procedure 1 (Table 1, entry 1): Under an Ar atmosphere, a $\mathrm{CH}_{2} \mathrm{Cl}_{2}(1.0 \mathrm{~mL})$ solution of H8-BINAP $(9.5 \mathrm{mg}, 0.015 \mathrm{mmol})$ was added to a $\mathrm{CH}_{2} \mathrm{Cl}_{2}(1.0 \mathrm{~mL})$ solution of $\left[\mathrm{Rh}(\operatorname{cod})_{2}\right] \mathrm{BF}_{4}(6.1 \mathrm{mg}, 0.015 \mathrm{mmol})$ at $\mathrm{rt}$. The solution was stirred at $\mathrm{rt}$ for $5 \mathrm{~min} . \mathrm{H}_{2}$ was introduced to the resulting solution in a Schlenk tube. After stirring at $\mathrm{rt}$ for $0.5 \mathrm{~h}$, the resulting solution was concentrated to dryness and dissolved in $\mathrm{CH}_{2} \mathrm{Cl}_{2}(0.5 \mathrm{~mL})$. This solution was added to a $\mathrm{CH}_{2} \mathrm{Cl}_{2}(0.5 \mathrm{~mL})$ solution of 4-octyne (2a, $33.0 \mathrm{mg}, 0.300$ $\mathrm{mmol})$ and washed remaining catalyst away by using $\mathrm{CH}_{2} \mathrm{Cl}_{2}(0.5 \mathrm{~mL})$. To this solution was added a $\mathrm{CH}_{2} \mathrm{Cl}_{2}(0.5 \mathrm{~mL})$ solution of diethyl acetylenedicarboxylate (1a, $102 \mathrm{mg}$, $0.600 \mathrm{mmol})$, and washed remaining substrate away by using $\mathrm{CH}_{2} \mathrm{Cl}_{2}(0.5 \mathrm{~mL})$. The solution was stirred at $\mathrm{rt}$ for $16 \mathrm{~h}$. The resulting solution was concentrated and purified by preparative TLC (hexane:ethyl acetate $=7: 3$ ), which furnished 5,6-dipropylphthalic acid diethyl ester (3aa, $102.3 \mathrm{mg}, 0.227 \mathrm{mmol}, 76 \%$ yield) as a colorless oil.

\section{5,6-Dipropylbenzene-1,2,3,4-tetracarboxylic acid tetraethyl ester (3aa).}<smiles>CCCCc1c(C(=O)OCC)c(C(=O)OCC)c(C(=O)OCC)c(C(=O)OCC)c1C(=O)OCC</smiles>

Colorless oil; IR (neat) 2920, 1720, 1190, 1100, $1015 \mathrm{~cm}^{-1} ;{ }^{1} \mathrm{H} \mathrm{NMR}\left(\mathrm{CDCl}_{3}, 300 \mathrm{MHz}\right)$ $\delta 4.35(\mathrm{q}, J=7.2 \mathrm{~Hz}, 4 \mathrm{H}), 4.28(\mathrm{q}, J=7.2 \mathrm{~Hz}, 4 \mathrm{H}), 2.63-2.69(\mathrm{~m}, 4 \mathrm{H}), 1.50-1.62(\mathrm{~m}$, $4 \mathrm{H}), 1.36(\mathrm{t}, J=7.2 \mathrm{~Hz}, 6 \mathrm{H}), 1.32(\mathrm{t}, J=7.2 \mathrm{~Hz}, 6 \mathrm{H}), 1.00(\mathrm{t}, J=7.2 \mathrm{~Hz}, 6 \mathrm{H}) ;{ }^{13} \mathrm{C} \mathrm{NMR}$ 
$\left(\mathrm{CDCl}_{3}, 75 \mathrm{MHz}\right) \delta 167.5,166.2,141.8,134.9,129.1,62.0,61.8,32.5,24.6,14.7,14.0$, 13.9; Anal calcd for $\mathrm{C}_{24} \mathrm{H}_{34} \mathrm{O}_{8}$ : C, 63.98; H, 7.61. Found: C, 64.13; H, 7.84.

5-Methyl-6-pentylbenzene-1,2,3,4-tetracarboxylic acid tetraethyl ester (3ba).<smiles>CCCc1c(C(=O)OCC)c(C(=O)OCC)c(C)c(C(=O)OCC)c1C(=O)OCC</smiles>

Colorless oil; IR (neat) 2910, 1720, 1230, $1020 \mathrm{~cm}^{-1} ;{ }^{1} \mathrm{H}$ NMR $\left(\mathrm{CDCl}_{3}, 300 \mathrm{MHz}\right)$ $\delta 4.37(\mathrm{q}, J=7.2 \mathrm{~Hz}, 2 \mathrm{H}), 4.36(\mathrm{q}, J=7.2 \mathrm{~Hz}, 2 \mathrm{H}), 4.28(\mathrm{q}, J=7.2 \mathrm{~Hz}, 4 \mathrm{H}), 2.63-2.70$ $(\mathrm{m}, 2 \mathrm{H}), 2.36(\mathrm{~s}, 3 \mathrm{H}), 1.46-1.58(\mathrm{~m}, 2 \mathrm{H}), 1.30-1.38(\mathrm{~m}, 16 \mathrm{H}), 0.90(\mathrm{t}, J=6.8 \mathrm{~Hz}, 3 \mathrm{H})$; ${ }^{13} \mathrm{C} \mathrm{NMR}\left(\mathrm{CDCl}_{3}, 75 \mathrm{MHz}\right) \delta 167.5,167.5,166.1,142.2,137.1,135.1,134.6,128.8$, 128.6, 61.9, 61.8, 61.7, 32.1, 31.2, 29.6, 22.3, 16.3, 14.01, 13.98, 13.9; Anal calcd for $\mathrm{C}_{24} \mathrm{H}_{34} \mathrm{O}_{8}: \mathrm{C}, 63.98 ; \mathrm{H}, 7.61$. Found: C, 64.10; H, 7.65.

6-Methylbiphenyl-2,3,4,5-tetracarboxylic acid tetraethyl ester (3ca).<smiles>CCOC(=O)c1c(C(=O)OCC)c(C(=O)OCC)c(-c2ccccc2)c(C(=O)OCC)c1C(=O)OCC</smiles>

Pale yellow oil; IR (neat) 2925, 1715, 1205, $1020 \mathrm{~cm}^{-1} ;{ }^{1} \mathrm{H}$ NMR $\left(\mathrm{CDCl}_{3}, 300 \mathrm{MHz}\right) \delta$ 7.34-7.44 (m, 3H), 7.13-7.20 (m, 2H), $4.38(\mathrm{q}, J=7.2 \mathrm{~Hz}, 2 \mathrm{H}), 4.33(\mathrm{q}, J=7.2 \mathrm{~Hz}, 2 \mathrm{H})$, $4.30(\mathrm{q}, J=7.2 \mathrm{~Hz}, 2 \mathrm{H}), 3.91(\mathrm{q}, J=7.2 \mathrm{~Hz}, 2 \mathrm{H}), 2.13(\mathrm{~s}, 3 \mathrm{H}), 1.37(\mathrm{t}, J=7.2 \mathrm{~Hz}, 3 \mathrm{H})$, $1.35(\mathrm{t}, J=7.2 \mathrm{~Hz}, 3 \mathrm{H}), 1.31(\mathrm{t}, J=7.2 \mathrm{~Hz}, 3 \mathrm{H}), 0.89(\mathrm{t}, J=7.2 \mathrm{~Hz}, 3 \mathrm{H}) ;{ }^{13} \mathrm{C}$ NMR $\left(\mathrm{CDCl}_{3}, 75 \mathrm{MHz}\right) \delta 167.4,166.8,166.1,165.9,142.8,137.5,137.1,135.3,134.9,130.2$, 128.9, 128.4, 128.2, 128.0, 62.1, 62.0, 61.9, 61.4, 17.7, 13.9, 13.8, 13.8, 13.4; Anal calad for $\mathrm{C}_{25} \mathrm{H}_{28} \mathrm{O}_{8}$ : C, 65.78; H, 6.18. Found: C, 65.50; H, 6.45.

6-Methylbenzene-1,2,3,4,5-pentacarboxylic acid pentaethyl ester (3da).<smiles>CCOC(=O)c1c(C(=O)OCC)c(C(=O)OCC)c(C(=O)OCC)c(C(=O)OCC)c1C(=O)OCC</smiles>

Colorless oil; IR (neat) 2940, 1720, 1210, $1015 \mathrm{~cm}^{-1} ;{ }^{1} \mathrm{H} \mathrm{NMR}\left(\mathrm{CDCl}_{3}, 300 \mathrm{MHz}\right)$ $\delta 4.38(\mathrm{q}, J=7.2 \mathrm{~Hz}, 4 \mathrm{H}), 4.32(\mathrm{q}, J=7.2 \mathrm{~Hz}, 6 \mathrm{H}), 2.41(\mathrm{~s}, 3 \mathrm{H}), 1.36(\mathrm{t}, J=7.2 \mathrm{~Hz}$, $6 \mathrm{H}), 1.34(\mathrm{t}, J=7.2 \mathrm{~Hz}, 9 \mathrm{H}) ;{ }^{13} \mathrm{C} \mathrm{NMR}\left(\mathrm{CDCl}_{3}, 75 \mathrm{MHz}\right) \delta 166.4,165.8,165.2,136.5$, 135.7, 131.2, 131.2, 62.3, 62.1, 62.1, 16.5, 13.9, 13.7; Anal calad for $\mathrm{C}_{22} \mathrm{H}_{28} \mathrm{O}_{10}$ : C, 58.40; H, 6.24. Found: C, 58.45; H, 6.41. 
Biphenyl-2,3,4,5,6-pentacarboxylic acid pentaethyl ester (3ea).<smiles>CCOC(=O)c1c(C(=O)OCC)c(C(=O)OCC)c(-c2ccccc2)c(C(=O)OCC)c1C(=O)OCC</smiles>

Colorless solid; Mp 84.0-85.6 ${ }^{\circ} \mathrm{C}$; IR (neat) 2920, 1720, 1200, $1010 \mathrm{~cm}^{-1}$; ${ }^{1} \mathrm{H}$ NMR $\left(\mathrm{CDCl}_{3}, 300 \mathrm{MHz}\right) \delta 7.32-7.40(\mathrm{~m}, 3 \mathrm{H}), 7.18-7.24(\mathrm{~m}, 2 \mathrm{H}), 4.35(\mathrm{q}, J=7.2 \mathrm{~Hz}, 2 \mathrm{H})$, $4.33(\mathrm{q}, J=7.2 \mathrm{~Hz}, 4 \mathrm{H}), 3.95(\mathrm{q}, J=7.2 \mathrm{~Hz}, 4 \mathrm{H}), 1.30-1.38(\mathrm{~m}, 9 \mathrm{H}), 0.87(\mathrm{t}, J=7.2 \mathrm{~Hz}$, $6 \mathrm{H}){ }^{13} \mathrm{C} \mathrm{NMR}\left(\mathrm{CDCl}_{3}, 75 \mathrm{MHz}\right) \delta 166.1,165.6,165.2,140.2,136.5,136.1,132.3$, 131.6, 128.5, 128.0, 62.5, 62.3, 61.7, 13.8, 13.7, 13.3; Anal calad for $\mathrm{C}_{27} \mathrm{H}_{30} \mathrm{O}_{10}$ : C, 63.03; H, 5.88. Found: C, 63.45; H, 5.97.

5,6-Bis(methoxymethyl)benzene-1,2,3,4-tetracarboxylic acid tetraethyl ester (3fa).<smiles>CCOC(=O)c1c(COC)c(COC)c(C(=O)OCC)c(C(=O)OCC)c1COC</smiles>

Colorless oil; IR (neat) 2890, 1720, 1215, 1095, $1015 \mathrm{~cm}^{-1} ;{ }^{1} \mathrm{H} \mathrm{NMR}\left(\mathrm{CDCl}_{3}, 300 \mathrm{MHz}\right)$ $\delta 4.63(\mathrm{~s}, 4 \mathrm{H}), 4.35(\mathrm{q}, J=7.2 \mathrm{~Hz}, 4 \mathrm{H}), 4.30(\mathrm{q}, J=7.0 \mathrm{~Hz}, 4 \mathrm{H}), 3.27(\mathrm{~s}, 6 \mathrm{H}), 1.36(\mathrm{t}, J$ $=7.2 \mathrm{~Hz}, 6 \mathrm{H}), 1.33(\mathrm{t}, J=7.2 \mathrm{~Hz}, 6 \mathrm{H}) ;{ }^{13} \mathrm{C} \mathrm{NMR}\left(\mathrm{CDC}_{3}, 75 \mathrm{MHz}\right) \delta 166.9,165.8$, $138.3,135.2,131.0,68.1,62.1,61.9,58.1,13.9,13.8$; Anal calad for $\mathrm{C}_{22} \mathrm{H}_{30} \mathrm{O}_{10}: \mathrm{C}$, 58.14; H, 6.65. Found: C, 58.15; H, 6.65 .

\section{Synthesis of Internal Alkynes (Tables 2 and 3)}

1-Methyl-2-prop-1-ynylbenzene (2h), ${ }^{1}$ 1-(3-methoxyprop-1-ynyl)-2-methylbenzene $(\mathbf{2 i}){ }^{2}$ and 4-(1-naphtyl)-3-butyn-1-ol (2p) ${ }^{3}$ were prepared according to the literatures.

Acetic acid 3-o-tolyl-prop-2-ynyl ester (2j).<smiles>CC(=O)OCC#Cc1ccccc1C</smiles>

This compound was prepared from 3-o-tolylprop-2-yn-1-ol ${ }^{4}$ by treatment with $\mathrm{Ac}_{2} \mathrm{O} / \mathrm{Et}_{3} \mathrm{~N}$. 
Pale yellow oil; IR (neat) 2900, 1740, 1215, 1025, $755 \mathrm{~cm}^{-1} ;{ }^{1} \mathrm{H}$ NMR $\left(\mathrm{CDCl}_{3}, 300\right.$ MHz) $\delta 7.42(\mathrm{~d}, J=7.5 \mathrm{~Hz}, 1 \mathrm{H}), 7.09-7.27(\mathrm{~m}, 3 \mathrm{H}), 4.94(\mathrm{~s}, 2 \mathrm{H}), 2.43(\mathrm{~s}, 3 \mathrm{H}), 2.14(\mathrm{~s}$, $3 \mathrm{H}) ;{ }^{13} \mathrm{C} \mathrm{NMR}\left(\mathrm{CDCl}_{3}, 75 \mathrm{MHz}\right) \delta 170.3,140.5,132.2,129.4,128.7,125.4,121.8$, 86.6, 85.3, 52.9, 20.8, 20.5; HRMS (EI) calcd for $\mathrm{C}_{12} \mathrm{H}_{12} \mathrm{O}_{2}[\mathrm{M}]^{+} 188.0837$, found 188.0812 .

\section{Propionic acid 3-o-tolyl-prop-2-ynyl ester (2k).}

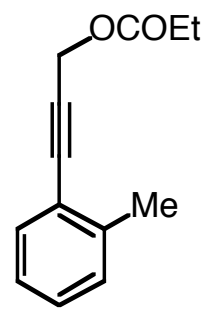

This compound was prepared from 3-o-tolylprop-2-yn-1-ol ${ }^{4}$ by treatment with $(\mathrm{EtCO})_{2} \mathrm{O} / \mathrm{Et}_{3} \mathrm{~N}$.

Colorless oil; IR (neat) 2900, 1735, 1160, $755 \mathrm{~cm}^{-1} ;{ }^{1} \mathrm{H}$ NMR $\left(\mathrm{CDCl}_{3}, 300 \mathrm{MHz}\right) \delta 7.41$ $(\mathrm{d}, J=7.5 \mathrm{~Hz}, 1 \mathrm{H}), 7.10-7.26(\mathrm{~m}, 3 \mathrm{H}), 4.95(\mathrm{~s}, 2 \mathrm{H}), 2.43(\mathrm{~s}, 3 \mathrm{H}), 2.41(\mathrm{q}, J=7.5 \mathrm{~Hz}$, $2 \mathrm{H}), 1.18(\mathrm{t}, J=7.5 \mathrm{~Hz}, 3 \mathrm{H}) ;{ }^{13} \mathrm{C} \mathrm{NMR}\left(\mathrm{CDCl}_{3}, 75 \mathrm{MHz}\right) \delta 173.7,140.5,132.1,129.4$, 128.7, 125.4, 121.9, 86.8, 85.2, 52.8, 27.4, 20.5, 9.0; HRMS (EI) calcd for $\mathrm{C}_{13} \mathrm{H}_{14} \mathrm{O}_{2}$ $[\mathrm{M}]^{+}$202.0994, found 202.0950.

Acetic acid 3-(2-ethylphenyl)prop-2-ynyl ester (2l).

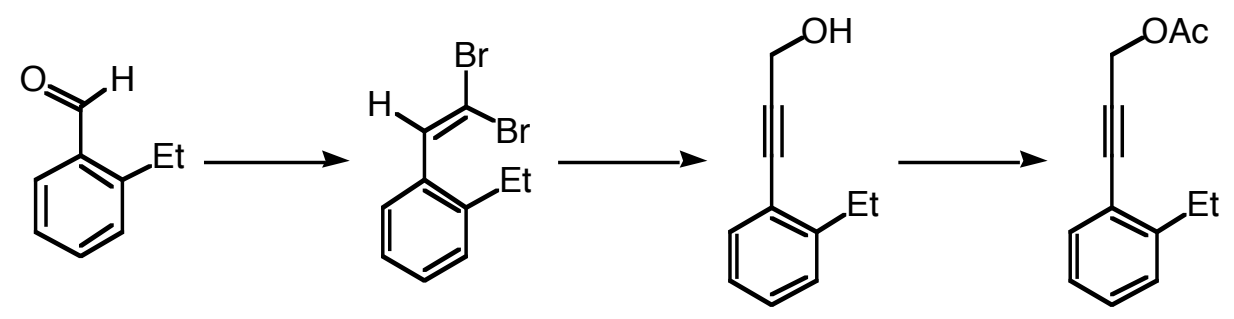

$\mathrm{CH}_{2} \mathrm{Cl}_{2}(100 \mathrm{~mL})$ solution of $\mathrm{PPh}_{3}(78.4 \mathrm{~g}, 0.298 \mathrm{~mol})$ was added to a stirred solution of $\mathrm{CBr}_{4}(49.4 \mathrm{~g}, 0.149 \mathrm{~mol})$ in $\mathrm{CH}_{2} \mathrm{Cl}_{2}(100 \mathrm{~mL})$ at $0{ }^{\circ} \mathrm{C}$, and the resulting mixture was stirred at $0{ }^{\circ} \mathrm{C}$ for $5 \mathrm{~min}$. $\mathrm{CH}_{2} \mathrm{Cl}_{2}(100 \mathrm{~mL})$ solution of 2-ethylbenzaldehyde $(5.00 \mathrm{~g}$, $37.3 \mathrm{mmol}$ ) was added at $0{ }^{\circ} \mathrm{C}$, and the resulting mixture was stirred at $0{ }^{\circ} \mathrm{C}$ for $20 \mathrm{~min}$. The reaction was diluted with water, and extracted with $\mathrm{CH}_{2} \mathrm{Cl}_{2}$. The organic layer was washed with saturated aqueous $\mathrm{NaHCO}_{3}$ and saturated aqueous $\mathrm{NH}_{4} \mathrm{Cl}$, dried over $\mathrm{Na}_{2} \mathrm{SO}_{4}$, and filtered. The filtrate was diluted with hexane and the resulting solid material was filtered. The filtrate was concentrated and the residue was purified by silica gel chromatography (hexanes:EtOAc $=10: 1)$ to give 1-(2,2-dibromovinyl)-2ethylbenzene ( $8.80 \mathrm{~g}, 30.4 \mathrm{mmol}, 81 \%$ yield $)$ as a brown oil.

${ }^{1} \mathrm{H}$ NMR $\left(\mathrm{CDCl}_{3}, 300 \mathrm{MHz}\right) \delta 7.52(\mathrm{~s}, 1 \mathrm{H}), 7.18-7.39(\mathrm{~m}, 4 \mathrm{H}), 2.57$ (q, $\left.J=7.5 \mathrm{~Hz}, 2 \mathrm{H}\right)$, $1.20(\mathrm{t}, J=7.5 \mathrm{~Hz}, 3 \mathrm{H})$.

$n$-BuLi (1.51 M in hexane, $24.0 \mathrm{~mL}, 36.2 \mathrm{mmol})$ was added to a stirred solution of 1(2,2-dibromovinyl)-2-ethylbenzene $(5.00 \mathrm{~g}, 17.2 \mathrm{mmol})$ in THF $(50 \mathrm{~mL})$ at $-70{ }^{\circ} \mathrm{C}$, and 
the resulting mixture was stirred at $-70{ }^{\circ} \mathrm{C}$ for $0.5 \mathrm{~h}$. Paraformaldehyde $(1.09 \mathrm{~g}, 0.362$ $\mathrm{mol}$ ) was added at $\mathrm{rt}$, and the resulting mixture was stirred at $\mathrm{rt}$ for $2 \mathrm{~h}$. The reaction was diluted with water, and extracted with $\mathrm{Et}_{2} \mathrm{O}$. The organic layer was washed with water and brine, dried over $\mathrm{Na}_{2} \mathrm{SO}_{4}$, and concentrated. The residue was purified by silica gel chromatography (hexanes:EtOAc $=10: 1)$ to give 3-(2-ethylphenyl)prop-2-yn-1-ol (2.50 $\mathrm{g}, 15.6 \mathrm{mmol}, 90 \%$ yield) as a brown oil.

${ }^{1} \mathrm{H} \mathrm{NMR}\left(\mathrm{CDCl}_{3}, 300 \mathrm{MHz}\right) \delta 7.41(\mathrm{~d}, J=7.2 \mathrm{~Hz}, 1 \mathrm{H}), 7.19-7.29(\mathrm{~m}, 2 \mathrm{H}), 7.11-7.16$ $(\mathrm{m}, 1 \mathrm{H}), 4.54(\mathrm{~d}, J=6.0 \mathrm{~Hz}, 2 \mathrm{H}), 2.80(\mathrm{q}, J=7.5 \mathrm{~Hz}, 2 \mathrm{H}), 1.78(\mathrm{t}, J=6.0 \mathrm{~Hz}, 1 \mathrm{H}), 1.23$ $(\mathrm{t}, J=7.5 \mathrm{~Hz}, 3 \mathrm{H})$.

$\mathrm{Ac}_{2} \mathrm{O}(0.500 \mathrm{~mL}, 6.24 \mathrm{mmol})$ was added to a stirred solution of 3-(2-ethylphenyl)prop2-yn-1-ol $(0.510 \mathrm{~g}, 3.18 \mathrm{mmol})$ and pyridine $(0.500 \mathrm{~mL}, 6.97 \mathrm{mmol})$ in $\mathrm{CH}_{2} \mathrm{Cl}_{2}(2.0$ $\mathrm{mL}$ ) at $\mathrm{rt}$, and the resulting mixture was stirred at $\mathrm{rt}$ for $3 \mathrm{~h}$. The reaction was diluted with water, and extracted with $\mathrm{Et}_{2} \mathrm{O}$. The organic layer was washed with brine, dried over $\mathrm{Na}_{2} \mathrm{SO}_{4}$, and concentrated. The residue was purified by silica gel chromatography (hexanes:EtOAc = 5:1) to give acetic acid 3-(2-ethylphenyl)prop-2-ynyl ester (2l, 0.639 g, $3.16 \mathrm{mmol}$, $99 \%$ yield) as a pale yellow oil.

Pale yellow oil; IR (neat) 3350, 2950, $174012201020 \mathrm{~cm}^{-1} ;{ }^{1} \mathrm{H}$ NMR (CDCl 3,300 MHz) $\delta 7.41(\mathrm{dd}, J=6.3$ and $1.2 \mathrm{~Hz}, 1 \mathrm{H}), 7.09-7.28(\mathrm{~m}, 3 \mathrm{H}), 4.93(\mathrm{~s}, 2 \mathrm{H}), 2.79(\mathrm{q}, J=$ $7.5 \mathrm{~Hz}, 2 \mathrm{H}), 2.11(\mathrm{~s}, 3 \mathrm{H}), 1.23(\mathrm{t}, J=7.5 \mathrm{~Hz}, 3 \mathrm{H}) ;{ }^{13} \mathrm{C} \mathrm{NMR}\left(\mathrm{CDCl}_{3}, 75 \mathrm{MHz}\right) \delta 170.2$, $146.5,132.4,128.9,127.8,125.5,121.1,86.2,85.0,52.8,27.5,20.7,14.7$; HRMS (EI) calcd for $\mathrm{C}_{13} \mathrm{H}_{14} \mathrm{O}_{2}[\mathrm{M}]^{+}$202.0944, found 202.1003.

Acetic acid 3-(2-chlorophenyl)prop-2-ynyl ester (2m).<smiles>CC(=O)OCC#Cc1ccccc1Cl</smiles>

This compound was prepared from 3-(2-chlorophenyl)prop-2-yn-1-ol ${ }^{4}$ by treatment with $\mathrm{Ac}_{2} \mathrm{O} / \mathrm{Et}_{3} \mathrm{~N}$.

Pale yellow oil; IR (neat) 2930, 1740, 1250, $1060750 \mathrm{~cm}^{-1} ;{ }^{1} \mathrm{H}$ NMR $\left(\mathrm{CDCl}_{3}, 300\right.$ MHz) $\delta 7.48(\mathrm{dd}, J=7.5$ and $2.1 \mathrm{~Hz}, 1 \mathrm{H}), 7.39(\mathrm{dd}, J=8.1$ and $1.2 \mathrm{~Hz}, 1 \mathrm{H}), 7.17-7.29$ $(\mathrm{m}, 2 \mathrm{H}), 4.95(\mathrm{~s}, 2 \mathrm{H}), 2.13(\mathrm{~s}, 3 \mathrm{H}) ;{ }^{13} \mathrm{C} \mathrm{NMR}\left(\mathrm{CDCl}_{3}, 75 \mathrm{MHz}\right) \delta 170.1,136.0,133.6$, 129.7, 129.2, 126.3 , 122.0, 88.0, 83.0, 52.6, 20.7; HRMS (EI) calcd for $\mathrm{C}_{11} \mathrm{H}_{9} \mathrm{ClO}_{2}[\mathrm{M}]^{+}$ 208.0291, found 208.0293.

Acetic acid 3-(2-bromophenyl)prop-2-ynyl ester (2n). 
<smiles>CC(=O)CC#Cc1ccccc1Br</smiles>

This compound was prepared from 3-(2-bromophenyl)prop-2-yn-1-ol ${ }^{4}$ by treatment with $\mathrm{Ac}_{2} \mathrm{O} / \mathrm{Et}_{3} \mathrm{~N}$.

Pale yellow oil; IR (neat) 2940, 1730, 1260, 1020, $750 \mathrm{~cm}^{-1} ;{ }^{1} \mathrm{H}$ NMR $\left(\mathrm{CDCl}_{3}, 300\right.$ MHz) $\delta 7.57(\mathrm{dd}, J=7.8$ and $1.2 \mathrm{~Hz}, 1 \mathrm{H}), 7.48(\mathrm{dd}, J=7.5$ and $1.8 \mathrm{~Hz} 1 \mathrm{H}), 7.15-7.28$ $(\mathrm{m}, 2 \mathrm{H}), 4.95(\mathrm{~s}, 2 \mathrm{H}), 2.14(\mathrm{~s}, 3 \mathrm{H}) ;{ }^{13} \mathrm{C} \mathrm{NMR}\left(\mathrm{CDCl}_{3}, 75 \mathrm{MHz}\right) \delta 170.2,133.7,132.4$, $129.9,126.9,125.5,124.2,87.5,84.7,52.7,20.7$; HRMS (EI) calcd for $\mathrm{C}_{11} \mathrm{H}_{9} \mathrm{BrO}_{2}[\mathrm{M}]^{+}$ 251.9786, found 251.9734 .

Acetic acid 3-naphthalen-1-yl-prop-2-ynyl ester (2o).<smiles>CC(=O)OCC#Cc1cccc2ccccc12</smiles>

This compound was prepared from 3-(naphthalen-1-yl)prop-2-yn-1-ol ${ }^{4}$ by treatment with $\mathrm{Ac}_{2} \mathrm{O} / \mathrm{Et}_{3} \mathrm{~N}$.

Pale yellow oil; IR (neat) 3050, 1740, 1210, 1010, $770 \mathrm{~cm}^{-1} ;{ }^{1} \mathrm{H}$ NMR $\left(\mathrm{CDCl}_{3}, 300\right.$ MHz) $\delta 8.31(\mathrm{~d}, J=9.0 \mathrm{~Hz}, 1 \mathrm{H}), 7.80(\mathrm{~d}, J=8.1 \mathrm{~Hz}, 2 \mathrm{H}), 7.68(\mathrm{dd}, J=7.2$ and $0.9 \mathrm{~Hz}$, $1 \mathrm{H}), 7.46-7.59(\mathrm{~m}, 2 \mathrm{H}), 7.38(\mathrm{dd}, J=8.1$ and $7.2 \mathrm{~Hz}, 1 \mathrm{H}), 5.04(\mathrm{~s}, 2 \mathrm{H}), 2.13(\mathrm{~s}, 3 \mathrm{H}) ;{ }^{13} \mathrm{C}$ NMR $\left(\mathrm{CDCl}_{3}, 75 \mathrm{MHz}\right) \delta 170.2$, 133.2, 133.0, 130.9, 129.2 128.2, 126.8, 126.4 125.9, 125.0, 119.6, 87.7 84.4, 52.9, 20.7; HRMS (EI) calcd for $\mathrm{C}_{15} \mathrm{H}_{12} \mathrm{O}_{2}[\mathrm{M}]^{+}$224.0837, found 224.0815 .

\section{Enantioselective Cross Cyclotrimerization of Dialkyl Acetylenedicarboxylate and Internal Alkynes (Tables 2 and 3)}

General Procedure 2 (Table 2, entry 3): Under an Ar atmosphere, a $\mathrm{CH}_{2} \mathrm{Cl}_{2}(1.0 \mathrm{~mL})$ solution of $(S)$-H8-BINAP $(9.5 \mathrm{mg}, 0.015 \mathrm{mmol})$ was added to a $\mathrm{CH}_{2} \mathrm{Cl}_{2}(1.0 \mathrm{~mL})$ solution of $\left[\mathrm{Rh}(\mathrm{cod})_{2}\right] \mathrm{BF}_{4}(6.1 \mathrm{mg}, 0.015 \mathrm{mmol})$ at $\mathrm{rt}$. The mixture was stirred at $\mathrm{rt}$ for 5 min. $\mathrm{H}_{2}$ was introduced to the resulting solution in a Schlenk tube. After stirring at $\mathrm{rt}$ for $0.5 \mathrm{~h}$, the resulting solution was concentrated to dryness and dissolved in $\mathrm{CH}_{2} \mathrm{Cl}_{2}(0.5$ $\mathrm{mL})$. This solution was added to a $\mathrm{CH}_{2} \mathrm{Cl}_{2}(0.5 \mathrm{~mL})$ solution of 3-tolyl-2-propynyl acetate $(2 \mathbf{j}, 56.4 \mathrm{mg}, 0.300 \mathrm{mmol})$ and washed remaining catalyst away by using $\mathrm{CH}_{2} \mathrm{Cl}_{2}$ $(0.5 \mathrm{~mL})$. To this solution was added a $\mathrm{CH}_{2} \mathrm{Cl}_{2}(0.5 \mathrm{~mL})$ solution of dimethyl acetylenedicarboxylate $(\mathbf{1 b}, 85.3 \mathrm{mg}, 0.600 \mathrm{mmol})$, and washed remaining substrate away by using $\mathrm{CH}_{2} \mathrm{Cl}_{2}(0.5 \mathrm{~mL})$. The solution was stirred at $\mathrm{rt}$ for $16 \mathrm{~h}$. The resulting 
solution was concentrated and purified by preparative TLC (hexane:ethyl acetate $=2: 1$ ), which furnished (+)-5-acetoxymethyl-6-tolylphthalic acid tetramethyl ester [(+)-3jb, $114.8 \mathrm{mg}, 0.243 \mathrm{mmol}, 81 \%$ yield, $89 \%$ ee] as a colorless solid.

(+)-6-Acetoxymethyl-2'-methylbiphenyl-2,3,4,5-tetracarboxylic acid tetramethyl ester $[(+)-3 \mathbf{j b}]$.<smiles>COC(=O)c1c(C(C)=O)c(C(C)=O)c(-c2ccccc2C)c(C(C)=O)c1C(=O)OC</smiles>

Colorless solid; $\mathrm{Mp} 144.0-144.5{ }^{\circ} \mathrm{C} ;[\alpha]_{\mathrm{D}}^{25}+27.5\left(\mathrm{CHCl}_{3}, c 0.754,89 \%\right.$ ee); IR (neat) 2950, 1720, 1440, 1340, $1220 \mathrm{~cm}^{-1} ;{ }^{1} \mathrm{H}$ NMR $\left(\mathrm{CDCl}_{3}, 300 \mathrm{MHz}\right) \delta 7.19-7.34(\mathrm{~m}, 3 \mathrm{H})$, $7.03(\mathrm{~d}, J=7.5 \mathrm{~Hz}, 1 \mathrm{H}), 4.89(\mathrm{~d}, J=12.6 \mathrm{~Hz}, 1 \mathrm{H}), 4.83(\mathrm{~d}, J=12.6 \mathrm{~Hz}, 1 \mathrm{H}), 3.91(\mathrm{~s}$, $3 \mathrm{H}), 3.90(\mathrm{~s}, 3 \mathrm{H}), 3.87(\mathrm{~s}, 3 \mathrm{H}), 3.45(\mathrm{~s}, 3 \mathrm{H}), 2.04(\mathrm{~s}, 3 \mathrm{H}), 1.95(\mathrm{~s}, 3 \mathrm{H}) ;{ }^{13} \mathrm{C} \mathrm{NMR}$ $\left(\mathrm{CDCl}_{3}, 75 \mathrm{MHz}\right) \delta 169.8,166.9,166.5,166.4,165.7,134.1,136.5,136.0,135.6,134.8$, 134.4, 131.7, 130.2, 129.9, 129.2, 129.0, 125.5, 60.7, 53.2, 53.0, 52.3, 20.3, 19.9; Anal calad for $\mathrm{C}_{24} \mathrm{H}_{24} \mathrm{O}_{10}$ : C, 61.01; H, 5.12. Found: C, 61.35; H, 5.08. CHIRALPAK AD, hexane: $i-\mathrm{PrOH}=80: 20,0.8 \mathrm{~mL} / \mathrm{min}$, retention times: $9.7 \mathrm{~min}$ (minor isomer) and 11.1 min (major isomer).

(-)-6,2'-Dimethylbiphenyl-2,3,4,5-tetracarboxylic acid tetramethyl ester [(-)-3hb].<smiles>COC(=O)c1c(C)c(-c2ccccc2C)c(C(C)=O)c(C(C)=O)c1C(C)=O</smiles>

Colorless oil; $[\alpha]^{25}{ }_{\mathrm{D}}-13.2\left(\mathrm{CHCl}_{3}, c 0.220,21 \%\right.$ ee); IR (neat) 2950, 1725, 1440, 1340, 1220, $730 \mathrm{~cm}^{-1}$; ${ }^{1} \mathrm{H}$ NMR $\left(\mathrm{CDCl}_{3}, 300 \mathrm{MHz}\right) \delta 7.18-7.32(\mathrm{~m}, 3 \mathrm{H}), 6.96(\mathrm{~d}, J=7.5 \mathrm{~Hz}$, $1 \mathrm{H}), 3.92(\mathrm{~s}, 3 \mathrm{H}), 3.89(\mathrm{~s}, 3 \mathrm{H}), 3.85(\mathrm{~s}, 3 \mathrm{H}), 3.43(\mathrm{~s}, 3 \mathrm{H}), 2.04(\mathrm{~s}, 3 \mathrm{H}), 2.03(\mathrm{~s}, 3 \mathrm{H}) ;{ }^{13} \mathrm{C}$ NMR $\left(\mathrm{CDCl}_{3}, 75 \mathrm{MHz}\right) \delta 167.7,167.0,166.7,166.2,142.9,138.4,136.3,136.2,135.3$, $134.7,130.5,129.9,128.6,128.5,128.1,125.7,53.1,53.0,52.8,52.2,19.7,17.3$; HRMS (FAB) calcd for $\mathrm{C}_{22} \mathrm{H}_{22} \mathrm{O}_{8}[\mathrm{M}+\mathrm{H}]^{+}$415.1393, found 415.1405. CHIRALPAK $\mathrm{AD}$, hexane: $i-\mathrm{PrOH}=90: 10,1.0 \mathrm{~mL} / \mathrm{min}$, retention times: $10.4 \mathrm{~min}$ (major isomer) and $11.6 \mathrm{~min}$ (minor isomer).

(+)-6-Acetoxymethyl-2'-methylbiphenyl-2,3,4,5-tetracarboxylic acid tetraethyl ester [(+)-3ja]. 
<smiles>CCOC(=O)c1c(C(=O)OCC)c(C(=O)OCC)c(-c2ccccc2C)c(C(=O)OCC)c1C(=O)OCC</smiles>

$(R)$-H8-BINAP was used as ligand. Colorless solid; Mp 79.5-80.9 ${ }^{\circ} \mathrm{C} ;[\alpha]^{25}{ }_{\mathrm{D}}+29.2^{\circ}$ $\left(\mathrm{CHCl}_{3}, c 1.01,93 \%\right.$ ee); IR (neat) 2940, 1716, 1420, 1365, 1215(br), 1020, $730 \mathrm{~cm}^{-1}$; ${ }^{1} \mathrm{H} \mathrm{NMR}\left(\mathrm{CDCl}_{3}, 300 \mathrm{MHz}\right) \delta 7.17-7.32(\mathrm{~m}, 3 \mathrm{H}), 7.04(\mathrm{~d}, J=7.2 \mathrm{~Hz}, 1 \mathrm{H}), 4.90(\mathrm{~d}, J=$ $12.0 \mathrm{~Hz}, 1 \mathrm{H}), 4.82(\mathrm{~d}, J=12.0 \mathrm{~Hz}, 1 \mathrm{H}), 4.24-4.39(\mathrm{~m}, 6 \mathrm{H}), 3.91(\mathrm{q}, J=7.2 \mathrm{~Hz}, 2 \mathrm{H})$, $2.06(\mathrm{~s}, 3 \mathrm{H}), 1.93(\mathrm{~s}, 3 \mathrm{H}), 1.37(\mathrm{t}, J=7.2 \mathrm{~Hz}, 3 \mathrm{H}), 1.36(\mathrm{t}, J=7.2 \mathrm{~Hz}, 3 \mathrm{H}), 1.32(\mathrm{t}, J=$ $7.2 \mathrm{~Hz}, 3 \mathrm{H}), 0.89(\mathrm{t}, J=7.2 \mathrm{~Hz}, 3 \mathrm{H}) ;{ }^{13} \mathrm{C} \mathrm{NMR}\left(\mathrm{CDCl}_{3}, 75 \mathrm{MHz}\right) \delta 169.8,166.5,166.2$, 166.0, 165.4, 142.8, 136.7, 135.9, 135.0, 134.7, 131.9, 130.7, 129.8, 129.4, 128.9, 125.4, 62.3, 62.2, 61.5, 60.7, 20.3, 20.0, 13.8, 13.7, 13.3; HRMS (FAB) calcd for $\mathrm{C}_{28} \mathrm{H}_{33} \mathrm{O}_{10}[\mathrm{M}+\mathrm{H}]^{+}$529.2074, found 529.2077. CHIRALPAK OD-H, hexane: $i-\mathrm{PrOH}=$ 95:5, $1.0 \mathrm{~mL} / \mathrm{min}$, retention times: $12.3 \mathrm{~min}$ (major isomer) and $14.0 \mathrm{~min}$ (minor isomer).

(+)-2'-Methyl-6-propionyloxymethylbiphenyl-2,3,4,5-tetracarboxylic acid tetramethyl ester [(+)-3kb].<smiles>CCOCc1c(COC)c(C(C)=O)c(C(C)=O)c(C(C)=O)c1-c1ccccc1C</smiles>

Colorless solid; $\mathrm{Mp} 89.6-90.8^{\circ} \mathrm{C}$; $[\alpha]^{25}{ }_{\mathrm{D}}+27.6^{\circ}\left(\mathrm{CHCl}_{3}, \mathrm{c} 0.984,91 \%\right.$ ee); IR (neat) 2940, 1725, 1215, $730 \mathrm{~cm}^{-1}$; ${ }^{1} \mathrm{H}$ NMR $\left(\mathrm{CDCl}_{3}, 300 \mathrm{MHz}\right) \delta 7.17-7.33(\mathrm{~m}, 3 \mathrm{H}), 7.03$ (dd, $J=7.2$ and $0.9 \mathrm{~Hz}, 1 \mathrm{H}), 4.90(\mathrm{~d}, J=12.3 \mathrm{~Hz}, 1 \mathrm{H}), 4.83(\mathrm{~d}, J=12.3 \mathrm{~Hz}, 1 \mathrm{H}), 3.91(\mathrm{~s}$, $3 \mathrm{H}), 3.89(\mathrm{~s}, 3 \mathrm{H}), 3.86(\mathrm{~s}, 3 \mathrm{H}), 3.44(\mathrm{~s}, 3 \mathrm{H}), 2.21(\mathrm{q}, J=7.5 \mathrm{~Hz}, 2 \mathrm{H}), 2.17(\mathrm{~s}, 3 \mathrm{H}), 2.04$ $(\mathrm{s}, 3 \mathrm{H}), 1.05(\mathrm{t}, J=7.5 \mathrm{~Hz}, 3 \mathrm{H}) ;{ }^{13} \mathrm{C} \mathrm{NMR}\left(\mathrm{CDCl}_{3}, 75 \mathrm{MHz}\right) \delta 173.2,166.9,166.5$, $166.4,165.7,143.1,136.5,135.9,135.8,134.8,134.5,131.7,130.2,129.9,129.2$, 129.0, 125.4, 60.5, 53.1, 52.9, 52.3, 27.0, 19.9, 8.8; Anal calad for $\mathrm{C}_{25} \mathrm{H}_{26} \mathrm{O}_{10}$ : C, 61.72; $\mathrm{H}$, 5.39. Found: $\mathrm{C}, 61.55 ; \mathrm{H}, 5.43$. CHIRALPAK AD, hexane: $i-\mathrm{PrOH}=92: 8,1.0$ $\mathrm{mL} / \mathrm{min}$, retention times: $20.5 \mathrm{~min}$ (minor isomer) and $24.8 \mathrm{~min}$ (major isomer).

(+)-6-Acetoxymethyl-2'-ethylbiphenyl-2,3,4,5-tetracarboxylic acid tetramethyl ester [(+)-3lb]. 


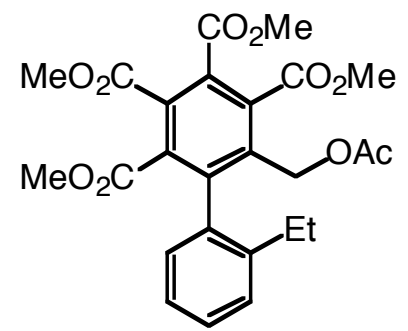

Colorless solid; $\mathrm{Mp} 46.0-48.2^{\circ} \mathrm{C} ;[\alpha]^{25}{ }_{\mathrm{D}}+15.1^{\circ}\left(\mathrm{CHCl}_{3}, c 1.05,92 \%\right.$ ee); IR (neat) 2925 , 1730, 1240, 1080, $1020 \mathrm{~cm}^{-1} ;{ }^{1} \mathrm{H}$ NMR $\left(\mathrm{CDCl}_{3}, 300 \mathrm{MHz}\right) \delta 7.29-7.39(\mathrm{~m}, 2 \mathrm{H}), 7.20$ (dt, $J=7.5$ and $1.5 \mathrm{~Hz}, 1 \mathrm{H}), 7.02(\mathrm{~d}, J=6.6 \mathrm{~Hz}, 1 \mathrm{H}), 4.89(\mathrm{~d}, J=12.6 \mathrm{~Hz}, 1 \mathrm{H}), 4.83(\mathrm{~d}$, $J=12.6 \mathrm{~Hz}, 1 \mathrm{H}), 3.91(\mathrm{~s}, 3 \mathrm{H}), 3.89(\mathrm{~s}, 3 \mathrm{H}), 3.86(\mathrm{~s}, 3 \mathrm{H}), 3.44(\mathrm{~s}, 3 \mathrm{H}), 2.24-2.38(\mathrm{~m}$, $2 \mathrm{H}), 1.94(\mathrm{~s}, 3 \mathrm{H}), 1.10(\mathrm{t}, J=7.5 \mathrm{~Hz}, 3 \mathrm{H}) ;{ }^{13} \mathrm{C} \mathrm{NMR}\left(\mathrm{CDCl}_{3}, 75 \mathrm{MHz}\right) \delta 169.7,166.9$, 166.5 166.4, 165.8, 143.0, 142.1, 136.0, 135.8, 134.7, 133.8, 131.7, 130.3, 129.4, 129.2, 127.8, 125.3, 60.8, 53.4, 53.2, 53.0, 52.3, 25.8, 20.4, 13.9; HRMS (FAB) calcd for $\mathrm{C}_{25} \mathrm{H}_{26} \mathrm{O}_{10}[\mathrm{M}+\mathrm{H}]^{+}$487.1604, found 487.1612. CHIRALPAK AD, Hexane: $i-\mathrm{PrOH}=$ $80: 20,0.8 \mathrm{~mL} / \mathrm{min}$, retention times: $8.9 \mathrm{~min}$ (minor isomer) and $10.4 \mathrm{~min}$ (major isomer).

(+)-6-Acetoxymethyl-2'-chlorobiphenyl-2,3,4,5-tetracarboxylic acid tetramethyl ester $[(+)-3 m b]$.<smiles>COC(=O)c1c(C(C)=O)c(C(C)=O)c(-c2ccccc2Cl)c(C(C)=O)c1C(=O)OC</smiles>

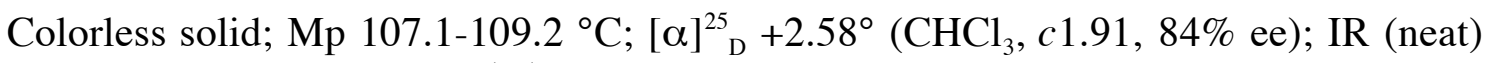
2910, 1720, 1440, $1220 \mathrm{~cm}^{-1} ;{ }^{1} \mathrm{H} \mathrm{NMR}\left(\mathrm{CDCl}_{3}, 300 \mathrm{MHz}\right) \delta 7.47(\mathrm{dd}, J=7.8$ and 1.5 $\mathrm{Hz}, 1 \mathrm{H}), 7.30-7.41(\mathrm{~m}, 2 \mathrm{H}), 7.23(\mathrm{dd}, J=7.2$ and $2.1 \mathrm{~Hz}, 1 \mathrm{H}), 4.96(\mathrm{~d}, J=12.6 \mathrm{~Hz}$, $1 \mathrm{H}), 4.84(\mathrm{~d}, J=12.6 \mathrm{~Hz}, 1 \mathrm{H}), 3.91(\mathrm{~s}, 6 \mathrm{H}), 3.87(\mathrm{~s}, 3 \mathrm{H}), 3.50(\mathrm{~s}, 3 \mathrm{H}), 1.93(\mathrm{~s}, 3 \mathrm{H}) ;{ }^{13} \mathrm{C}$ NMR $\left(\mathrm{CDCl}_{3}, 75 \mathrm{MHz}\right) \delta 169.8,166.7,166.2,166.1,165.6,140.7,136.0,135.5,134.9$, 134.0, 133.6, 132.2, 131.1, 130.8, 130.4, 129.3, 126.4, 60.6, 53.2, 53.0, 52.5, 20.3; HRMS (ESI) calcd for $\mathrm{C}_{23} \mathrm{H}_{21} \mathrm{ClO}_{10}[\mathrm{M}+\mathrm{Na}]^{+}$515.0721, found 515.0711. CHIRALPAK $\mathrm{AD}$, hexane: $i-\mathrm{PrOH}$ 80:20, $0.8 \mathrm{~mL} / \mathrm{min}$, retention times: $14.3 \mathrm{~min}$ (minor isomer) and 16.2 min (major isomer).

(R)-(-)-6-Acetoxymethyl-2'-bromobiphenyl-2,3,4,5-tetracarboxylic acid tetramethyl ester $[(R)-(-)-3 n b]$. 


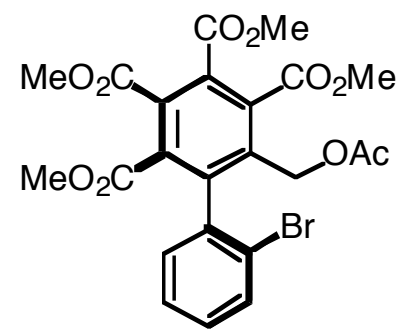

Colorless solid; Mp 115.0-117.0 ${ }^{\circ} \mathrm{C} ;[\alpha]^{25}{ }_{\mathrm{D}}-6.09^{\circ}\left(\mathrm{CHCl}_{3}, c 1.61,91 \%\right.$ ee); IR (neat) 2930, 1720, 1430, 1210, $1020 \mathrm{~cm}^{-1}$; ${ }^{1} \mathrm{H}$ NMR $\left(\mathrm{CDCl}_{3}, 300 \mathrm{MHz}\right) \delta 7.64(\mathrm{~d}, J=7.8 \mathrm{~Hz}$, $1 \mathrm{H}), 7.22-7.41(\mathrm{~m}, 3 \mathrm{H}), 4.97(\mathrm{~d}, J=12.6 \mathrm{~Hz}, 1 \mathrm{H}), 4.82(\mathrm{~d}, J=12.6 \mathrm{~Hz}, 1 \mathrm{H}), 3.91(\mathrm{~s}$, $6 \mathrm{H}), 3.87(\mathrm{~s}, 3 \mathrm{H}), 3.50(\mathrm{~s}, 3 \mathrm{H}), 1.94(\mathrm{~s}, 3 \mathrm{H}){ }^{13} \mathrm{C} \mathrm{NMR}\left(\mathrm{CDCl}_{3}, 75 \mathrm{MHz}\right) \delta 169.8,166.7$, $166.3,166.0,165.7,142.3,136.1,135.8,135.3,134.9,132.5,132.2,131.2,130.8$, $130.4,126.9,123.6,60.7,53.2,53.0,52.5,20.4$; HRMS (ESI) calcd for $\mathrm{C}_{23} \mathrm{H}_{21} \mathrm{BrO}_{10}$ $[\mathrm{M}+\mathrm{Na}]^{+}$559.0216, found 559.0223. CHIRALPAK AD, hexane: $i$-PrOH $=70: 30,0.8$ $\mathrm{mL} / \mathrm{min}$, retention times: $9.6 \mathrm{~min}$ (minor isomer) and $11.2 \mathrm{~min}$ (major isomer).

(+)-5-Acetoxymethyl-6-naphthalen-1-yl-benzene-1,2,3,4-tetracarboxylic acid tetramethyl ester $[(+)-30 b]$.<smiles>COC(=O)c1c(C(C)=O)c(C(C)=O)c(COC(C)=O)c(-c2cccc3ccccc23)c1C(C)=O</smiles>

Pale yellow oil; $[\alpha]^{25}+9.30^{\circ}\left(\mathrm{CHCl}_{3}, c 1.93,95 \%\right.$ ee); IR (neat) $2925,1720,1440,1240$ $\mathrm{cm}^{-1} ;{ }^{1} \mathrm{H} \mathrm{NMR}\left(\mathrm{CDCl}_{3}, 300 \mathrm{MHz}\right) \delta 7.89(\mathrm{t}, J=8.4 \mathrm{~Hz}, 2 \mathrm{H}), 7.38-7.53(\mathrm{~m}, 3 \mathrm{H}), 7.26-$ $7.32(\mathrm{~m}, 2 \mathrm{H}), 4.80(\mathrm{~d}, J=12.3 \mathrm{~Hz}, 1 \mathrm{H}), 4.76(\mathrm{~d}, J=12.3 \mathrm{~Hz}, 1 \mathrm{H}), 3.93(\mathrm{~s}, 3 \mathrm{H}), 3.90(\mathrm{~s}$, $3 \mathrm{H}), 3.86(\mathrm{~s}, 3 \mathrm{H}), 3.21(\mathrm{~s}, 3 \mathrm{H}), 1.76(\mathrm{~s}, 3 \mathrm{H}) ;{ }^{13} \mathrm{C} \mathrm{NMR}\left(\mathrm{CDCl}_{3}, 100 \mathrm{MHz}\right) \delta 169.6$ 166.9, 166.4, 166.8, 142.1, 136.6, 135.0133 .1 132.4, 131.9, 131.8, 130.7, 129.3, 128.2, 127.5, 126.6, 126.2, 125.6, 124.8, 60.8, 53.2, 53.2, 53.0, 52.2, 20.1; HRMS (FAB) calcd for $\mathrm{C}_{27} \mathrm{H}_{24} \mathrm{O}_{10}[\mathrm{M}]^{+}$508.1370, found 508.1366. CHIRALPAK AD, hexane: $i-\mathrm{PrOH}=$ 80:20, $0.8 \mathrm{~mL} / \mathrm{min}$, retention times: $16.3 \mathrm{~min}$ (major isomer) and $19.3 \mathrm{~min}$ (minor isomer).

(+)-5-(2-Acetoxyethyl)-6-naphthalen-1-yl-benzene-1,2,3,4-tetracarboxylic acid tetraethyl ester [(+)-3pa]. Under an Ar atmosphere, a $\mathrm{CH}_{2} \mathrm{Cl}_{2}(1.0 \mathrm{~mL})$ solution of $(S)$ H8-BINAP (9.5 mg, $0.015 \mathrm{mmol})$ was added to a $\mathrm{CH}_{2} \mathrm{Cl}_{2}(1.0 \mathrm{~mL})$ solution of $\left[\mathrm{Rh}(\mathrm{cod})_{2}\right] \mathrm{BF}_{4}(6.1 \mathrm{mg}, 0.015 \mathrm{mmol})$ at $\mathrm{rt}$. The mixture was stirred at $\mathrm{rt}$ for $5 \mathrm{~min} . \mathrm{H}_{2}$ was introduced to the resulting solution in a Schlenk tube. After stirring at $\mathrm{rt}$ for $0.5 \mathrm{~h}$, the resulting solution was concentrated to dryness and dissolved in $\mathrm{CH}_{2} \mathrm{Cl}_{2}(0.5 \mathrm{~mL})$. This solution was added to a $\mathrm{CH}_{2} \mathrm{Cl}_{2}(0.5 \mathrm{ml})$ solution of 4-(1-naphtyl)-3-butyn-1-ol (59.5 mg, $0.300 \mathrm{mmol}$ ) and washed remaining catalyst away by using $\mathrm{CH}_{2} \mathrm{Cl}_{2}(0.5 \mathrm{~mL})$. To this solution was added a $\mathrm{CH}_{2} \mathrm{Cl}_{2}(0.5 \mathrm{ml})$ solution of diethyl acetylenedicarboxylate 
(102 $\mathrm{mg}, 0.600 \mathrm{mmol}$ ), and washed remaining substrate away by using $\mathrm{CH}_{2} \mathrm{Cl}_{2}(0.5$ $\mathrm{mL})$. The solution was stirred at $\mathrm{rt}$ for $16 \mathrm{~h}$. The resulting solution was diluted with $\mathrm{CH}_{2} \mathrm{Cl}_{2}(5.0 \mathrm{~mL})$. To this solution, $\mathrm{Ac}_{2} \mathrm{O}(0.28 \mathrm{ml}, 3.0 \mathrm{mmol})$ and $\mathrm{Et}_{3} \mathrm{~N}(0.42 \mathrm{ml}, 3.0$ $\mathrm{mmol}$ ) was added at $\mathrm{rt}$ and the solution was stirring at $\mathrm{rt}$ for $3 \mathrm{~h}$. The resulting solution was concentrated and purified by preparative TLC (hexane:ethyl acetate $=2: 1$ ), which furnished (+)-5-(2-acetoxyethyl)-6-naphthalen-1-yl-benzene-1,2,3,4-tetracarboxylic acid tetraethyl ester [(+)-3pa, $130.1 \mathrm{mg}, 0.225 \mathrm{mmol}, 75 \%$ yield, $96 \%$ ee] as a pale yellow solid.<smiles>CCOC(=O)c1c(CCOC(C)=O)c(-c2cccc3ccccc23)c(C(=O)OCC)c(C(=O)OCC)c1C(=O)OCC</smiles>

Pale yellow solid; Mp 43.2-44.8 ${ }^{\circ} \mathrm{C} ;[\alpha]^{25}{ }_{\mathrm{D}}+10.7\left(\mathrm{CHCl}_{3}, c 0.754,96 \%\right.$ ee); IR (neat) 2950, 1720, 1220, $1020 \mathrm{~cm}^{-1} ;{ }^{1} \mathrm{H} \mathrm{NMR}\left(\mathrm{CDCl}_{3}, 300 \mathrm{MHz}\right) \delta 7.90(\mathrm{t}, J=8.4 \mathrm{~Hz}, 2 \mathrm{H})$, 7.26-7.55 (m, 5H), 4.27-4.44 (m, 6H), 3.94-4.02 (m, 1H), 3.83-3.91 (m, 1H), 3.63 (q, $J$ $=6.9 \mathrm{~Hz}, 2 \mathrm{H}), 2.83-2.93(\mathrm{~m}, 1 \mathrm{H}), 2.55-2.64(\mathrm{~m}, 1 \mathrm{H}), 1.84(\mathrm{~s}, 3 \mathrm{H}), 1.39(\mathrm{t}, J=7.2 \mathrm{~Hz}$, $3 \mathrm{H}), 1.38(\mathrm{t}, J=7.2 \mathrm{~Hz}, 3 \mathrm{H}), 1.31(\mathrm{t}, J=7.2 \mathrm{~Hz}, 3 \mathrm{H}), 0.42(\mathrm{t}, J=7.2 \mathrm{~Hz}, 3 \mathrm{H}) ;{ }^{13} \mathrm{C} \mathrm{NMR}$ $\left(\mathrm{CDCl}_{3}, 75 \mathrm{MHz}\right) \delta 170.3,167.0,166.3,166.2,165.7,141.7,138.2,136.7,135.4,133.4$, 133.2, 131.9, 131.8, 129.6, 129.1, 128.2, 127.9, 126.7, 126.2, 125.7, 124.8, 63.4, 62.33, 62.30, 62.26, 61.2, 30.4, 20.7, 13.88, 13.86, 13.8, 12.9; HRMS (FAB) calcd for $\mathrm{C}_{32} \mathrm{H}_{34} \mathrm{O}_{10}[\mathrm{M}]^{+}$578.2152, found 578.2137. CHIRALPAK AD, hexane: $i$-PrOH = 80:20, $0.8 \mathrm{~mL} / \mathrm{min}$, retention times: $10.4 \mathrm{~min}$ (minor isomer) and $15.6 \mathrm{~min}$ (major isomer).

\section{References}

1) Weiss, H. M.; Touchette, K. M.; Angell, S.; Khan, J. Org. Biomol. Chem. 2003, 1, 2152-2156.

2) Russell, C. E.; Hegedus, L. S. J. Am. Chem. Soc. 1983, 105, 943-949.

3) Feuerstein, M.; Berthiol, F.; Doucet, H.; Santelli, M. Synthesis 2004, 1281-1289.

4) Liron, F.; Le Garrec, P.; Alami, M. Synlett 1999, 246-248. 

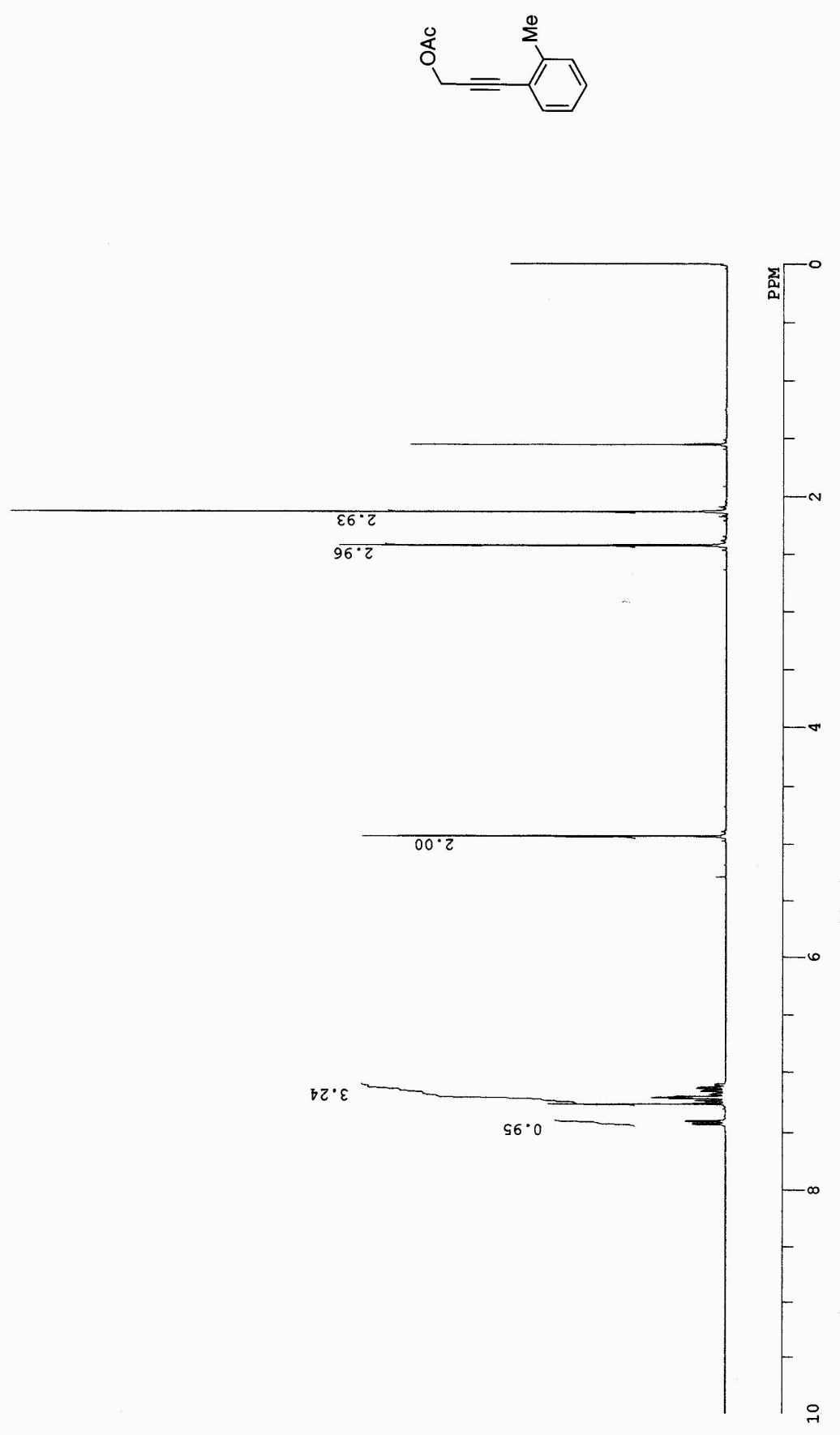
$\underbrace{+1}_{=1}$

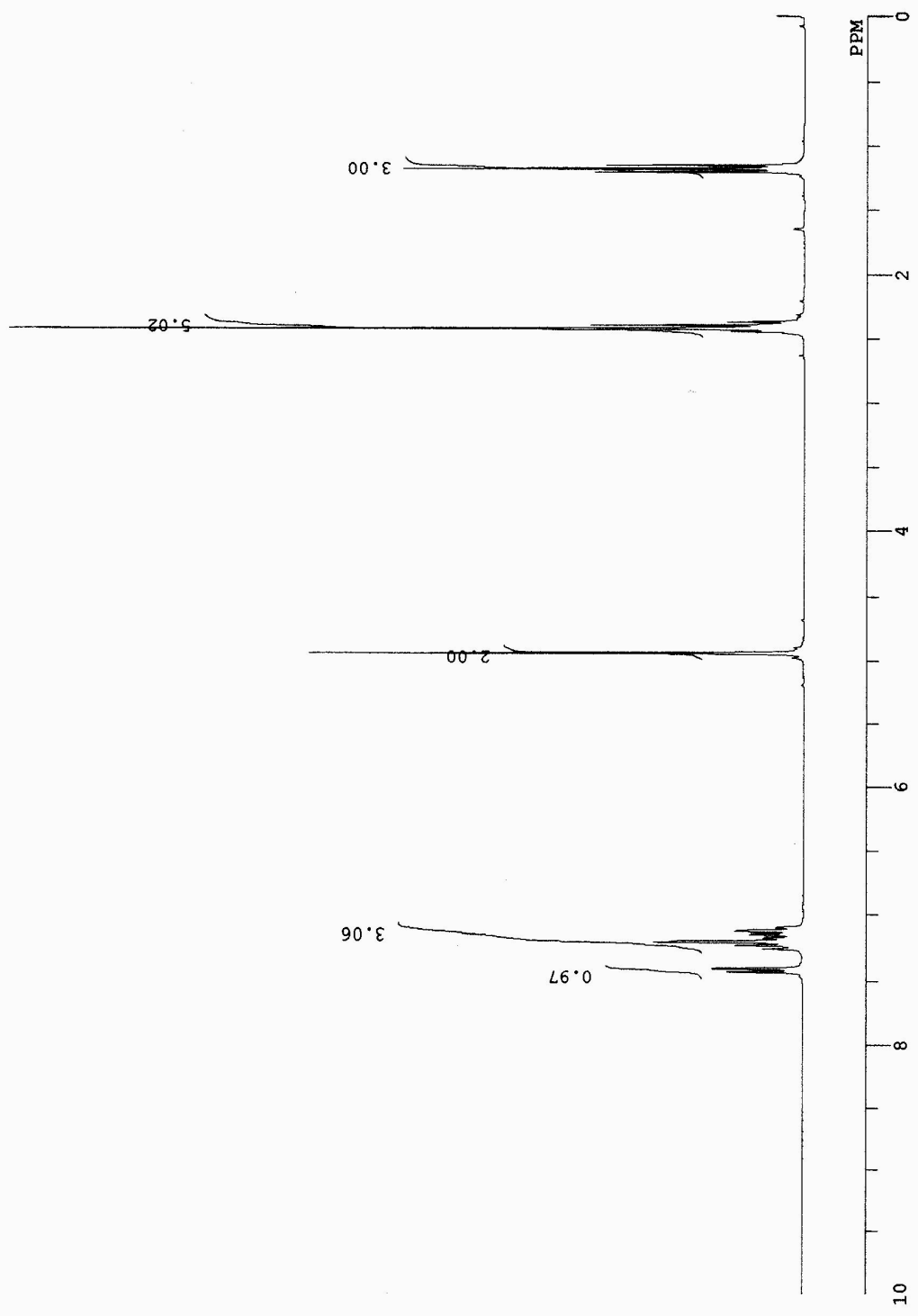



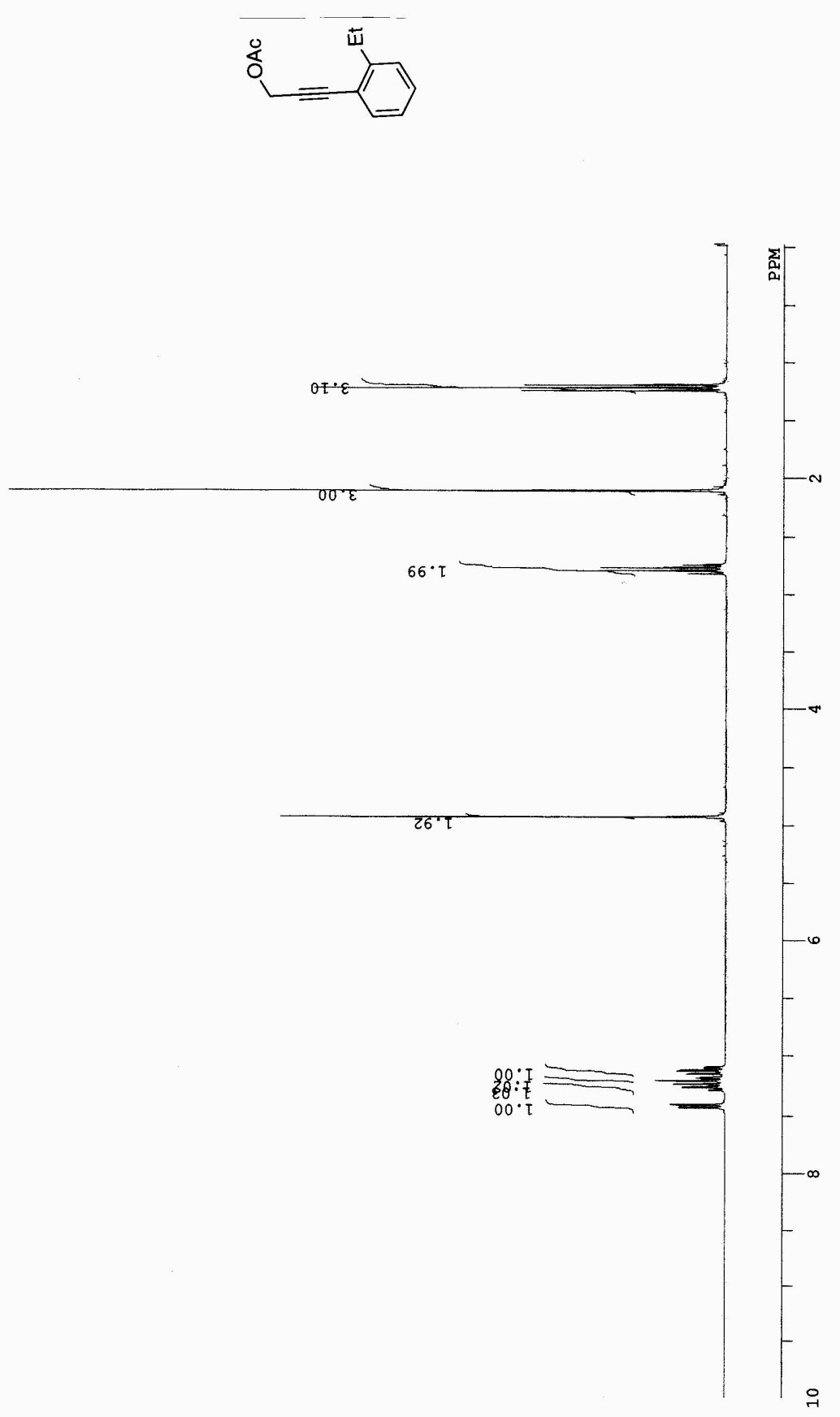

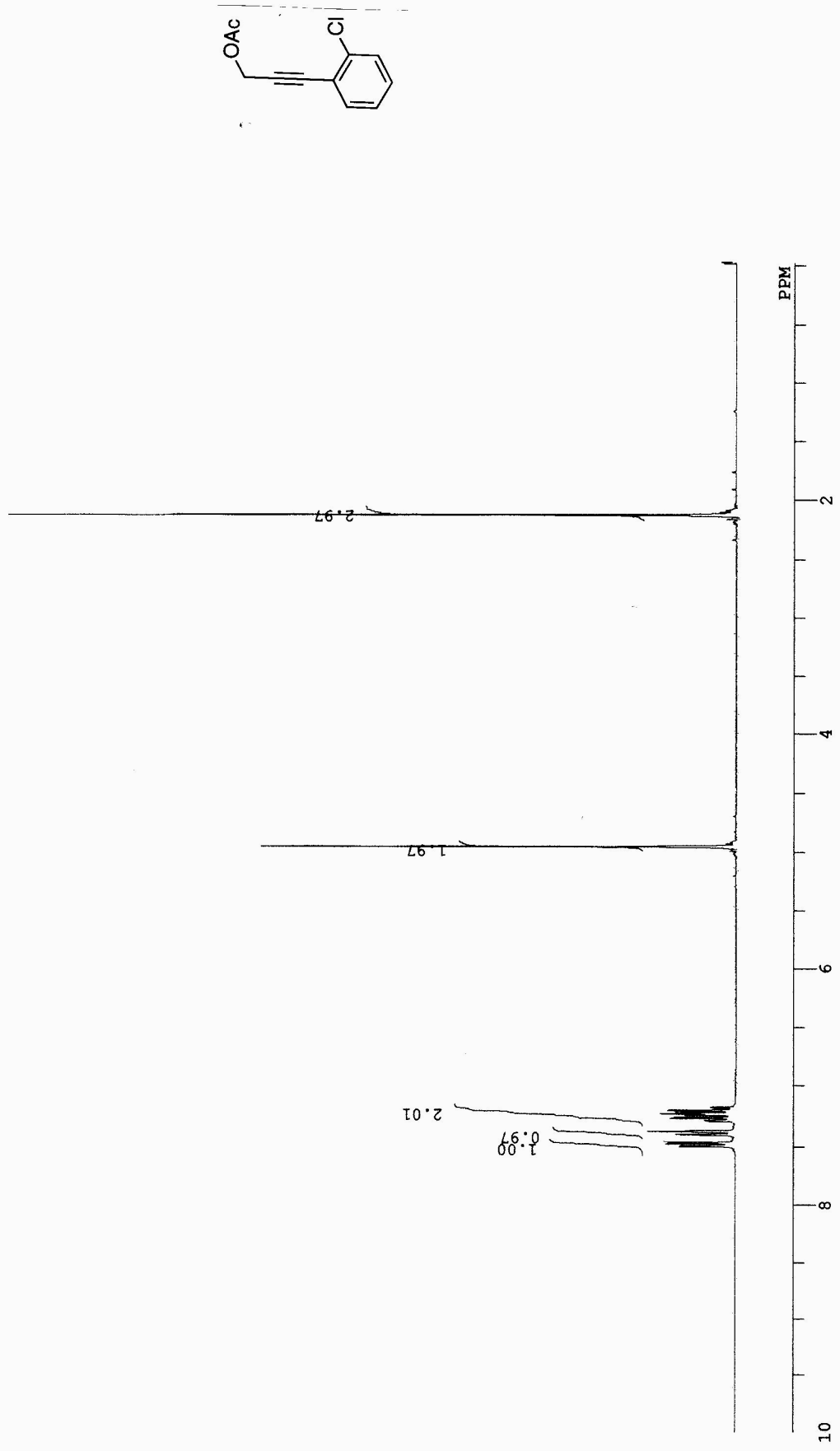

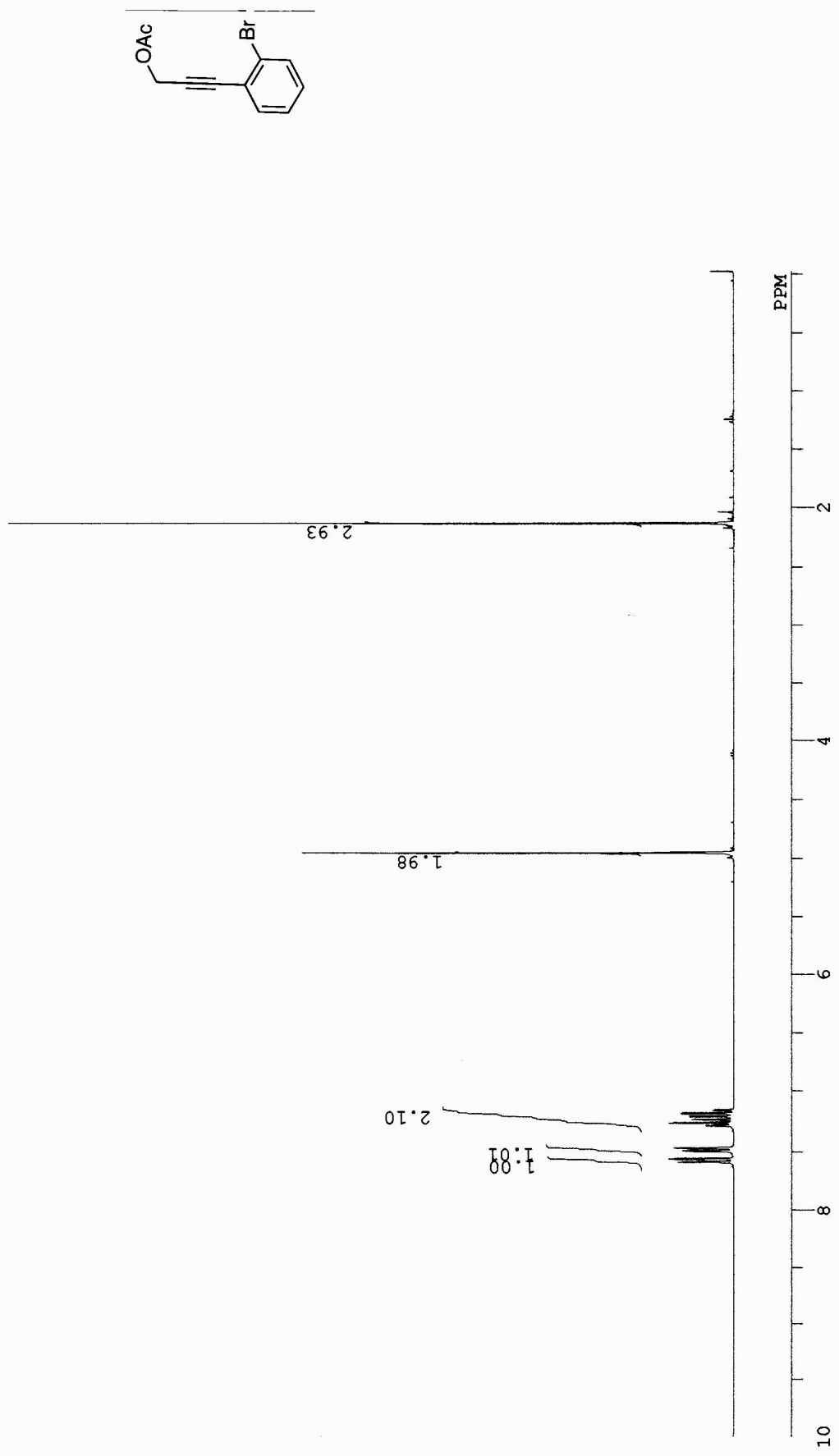

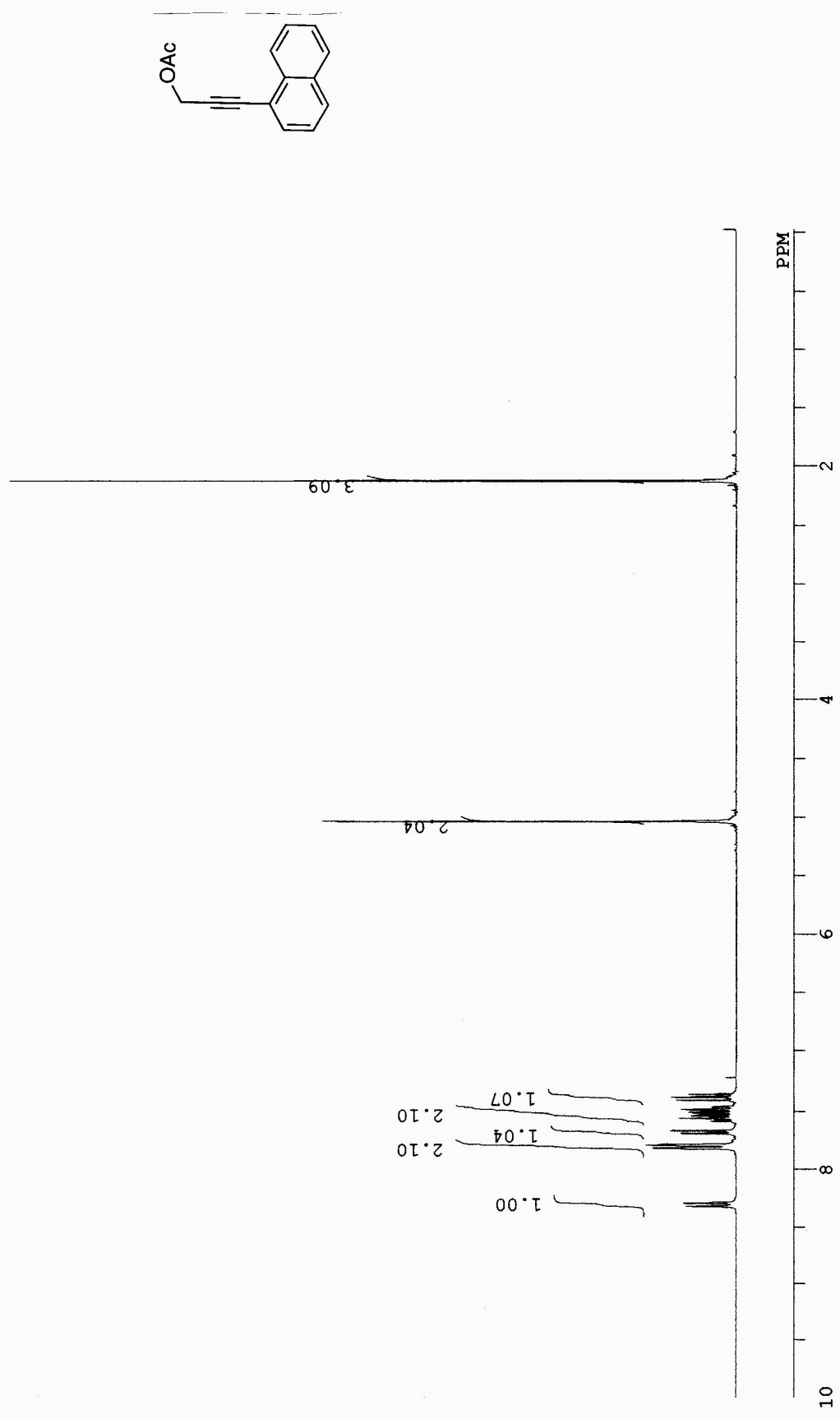

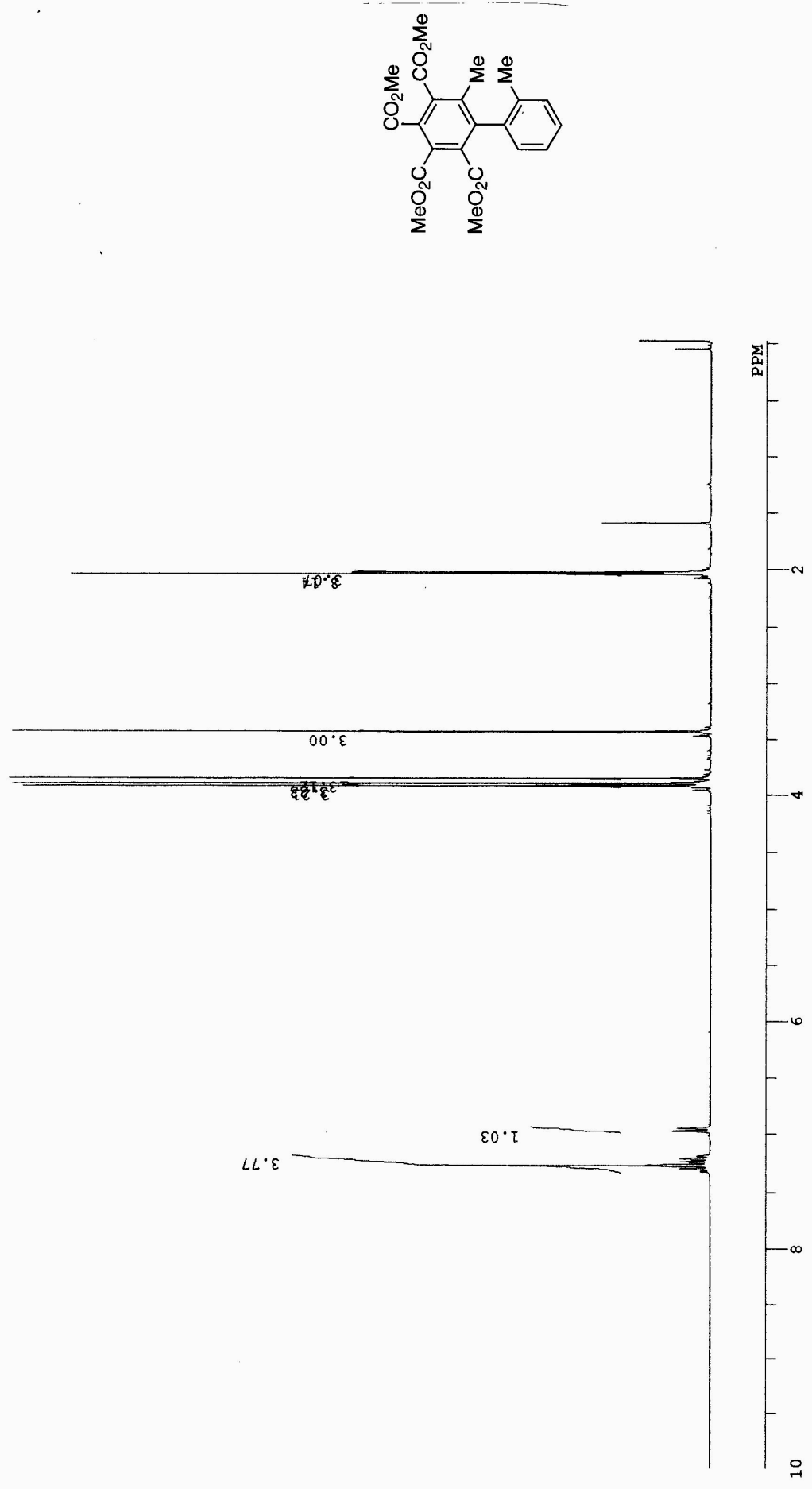

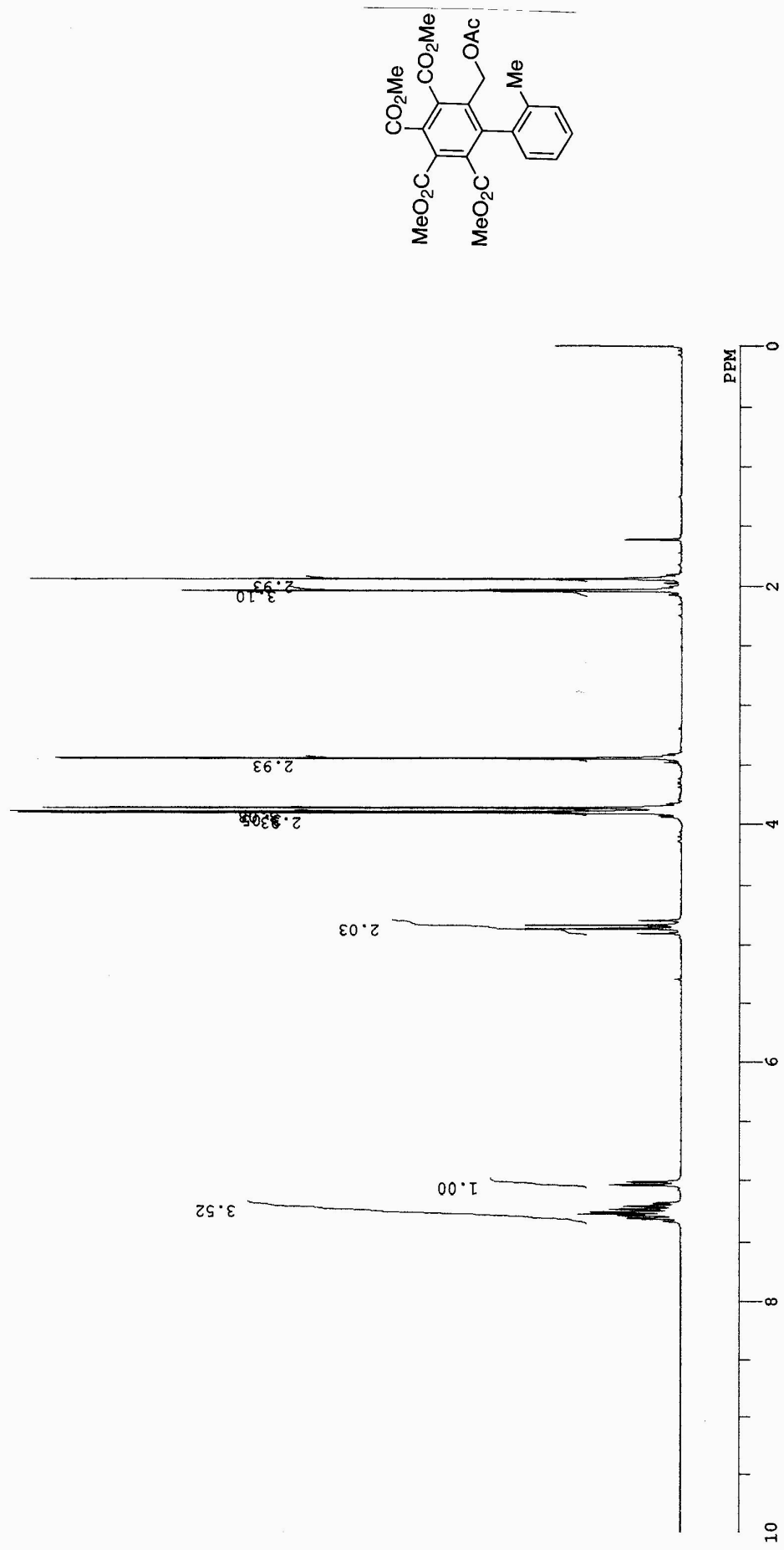

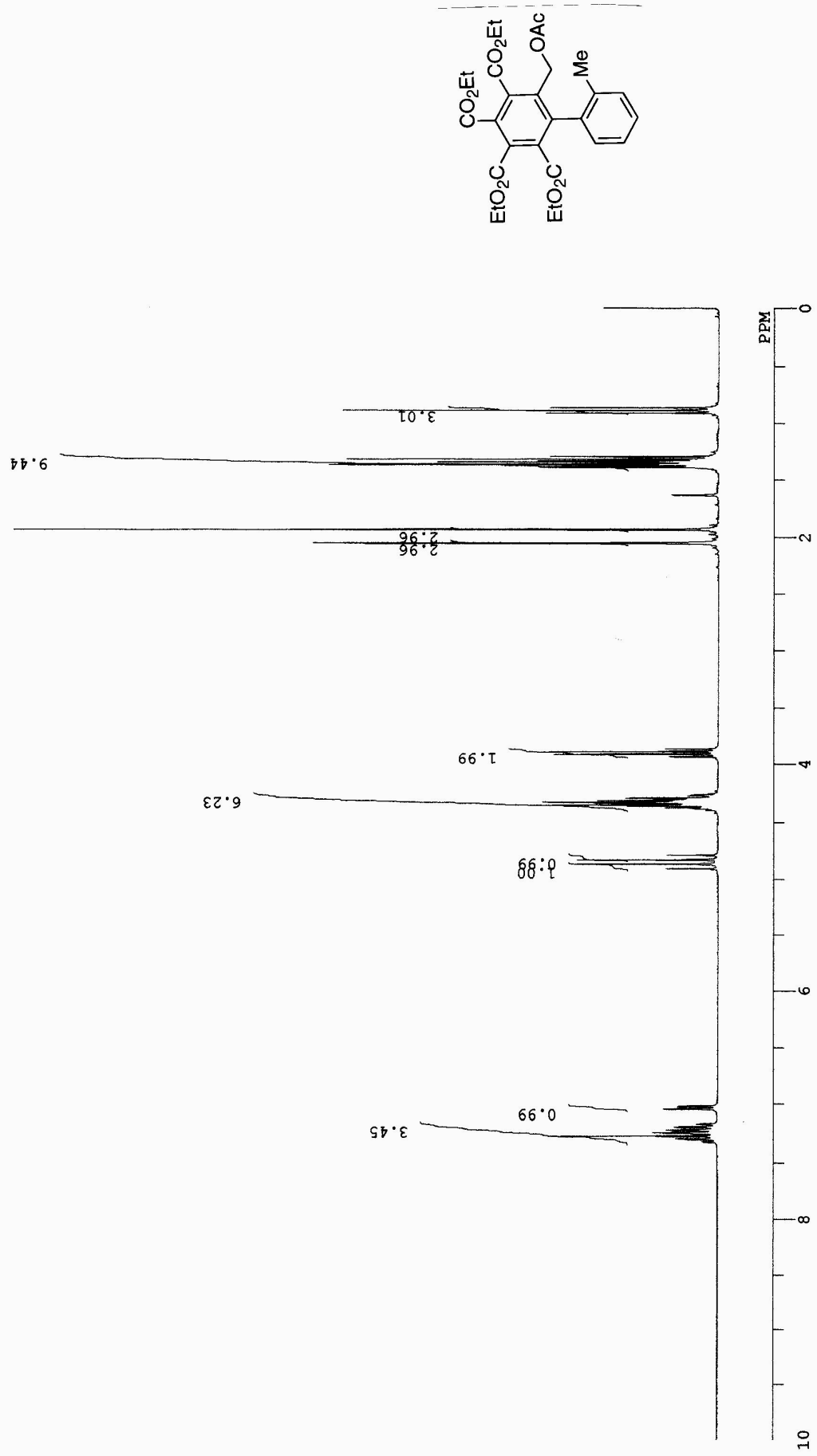

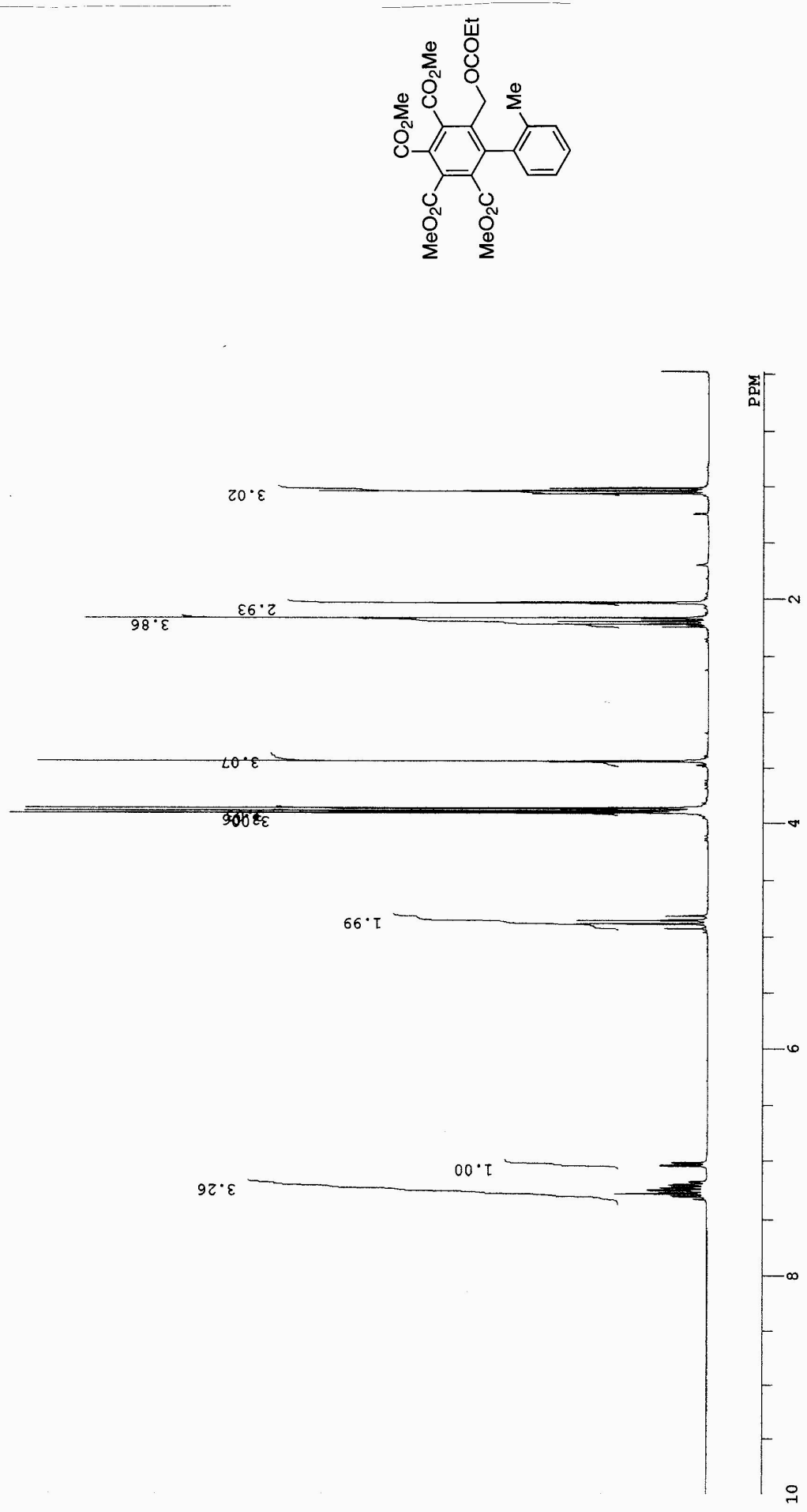

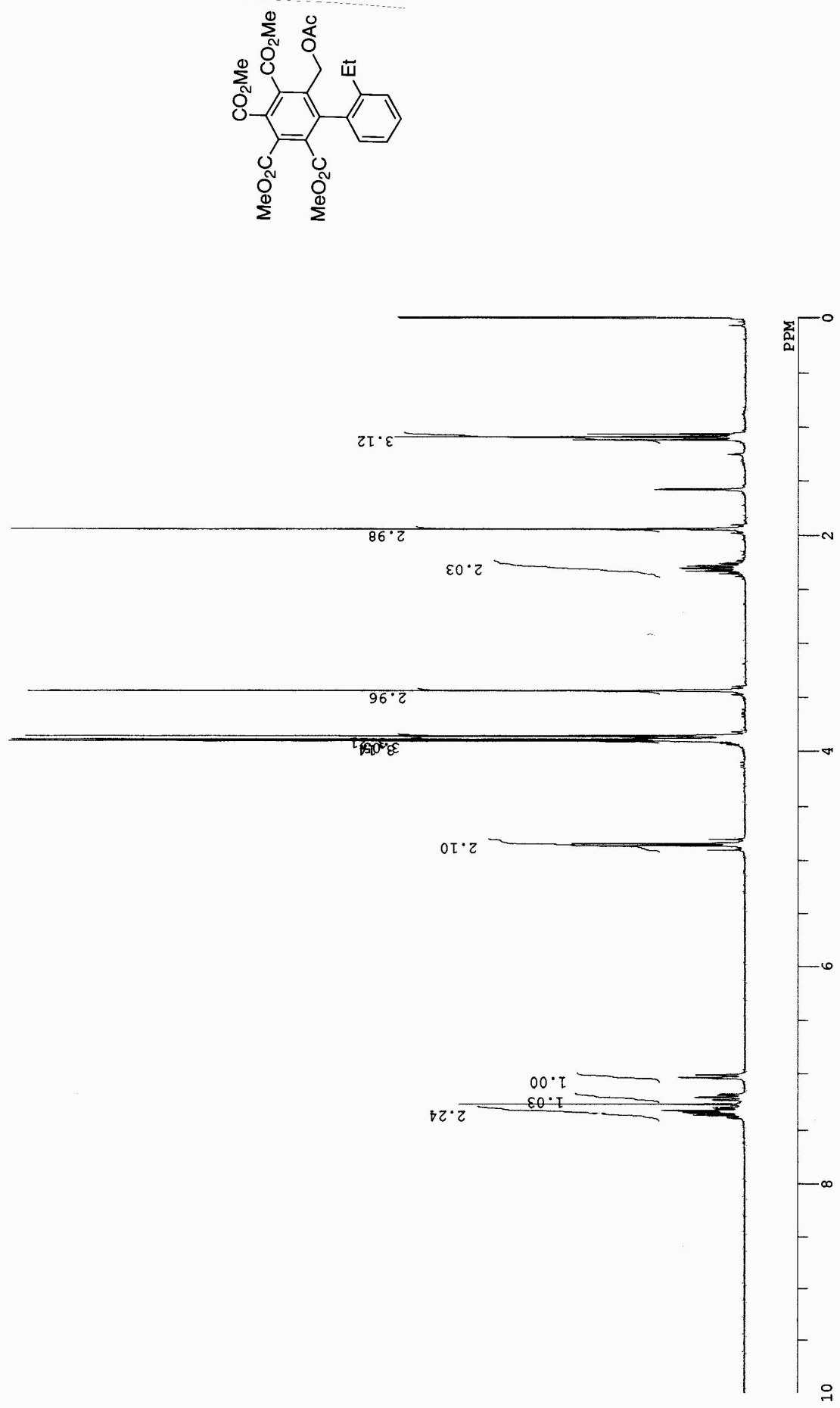

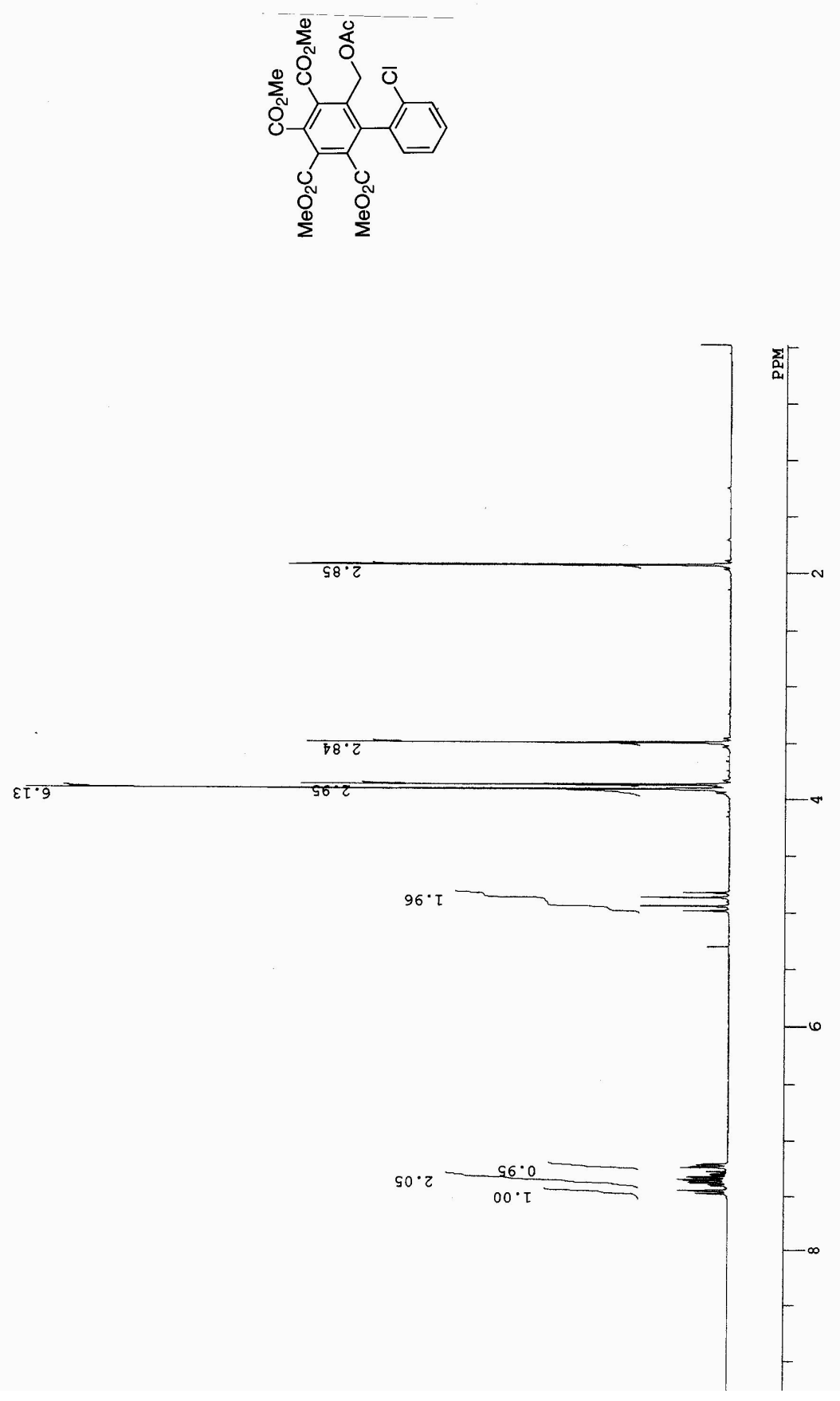

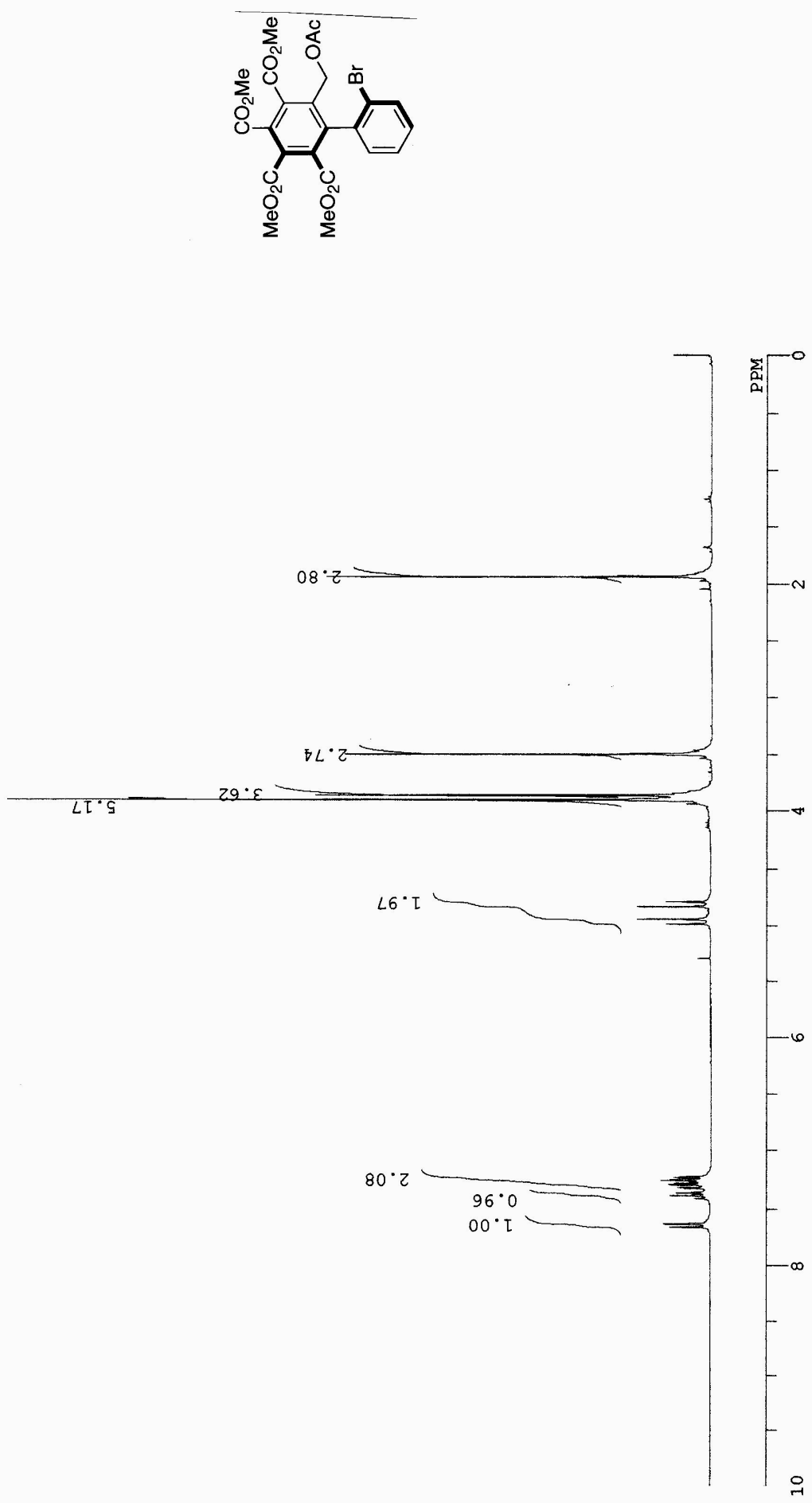

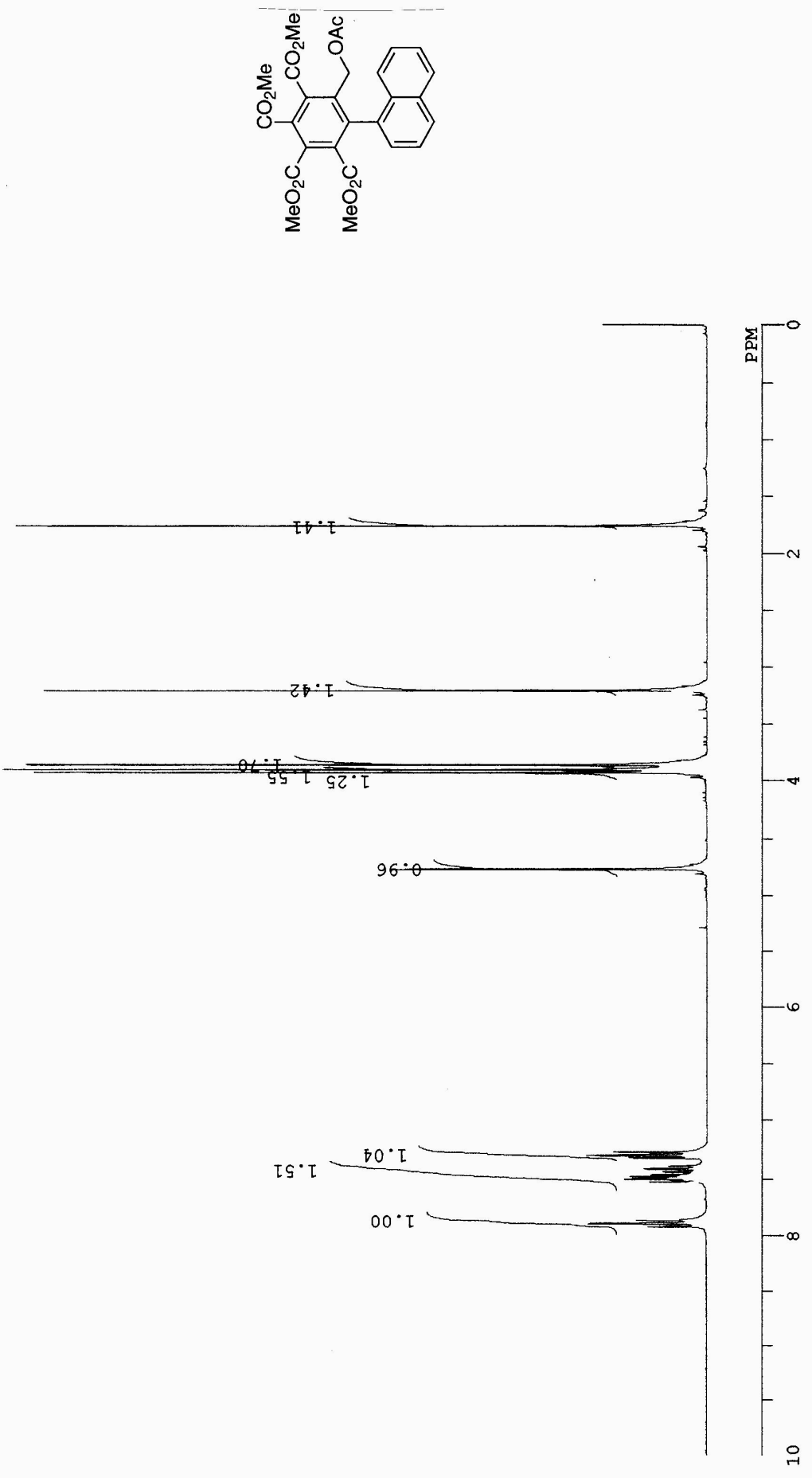

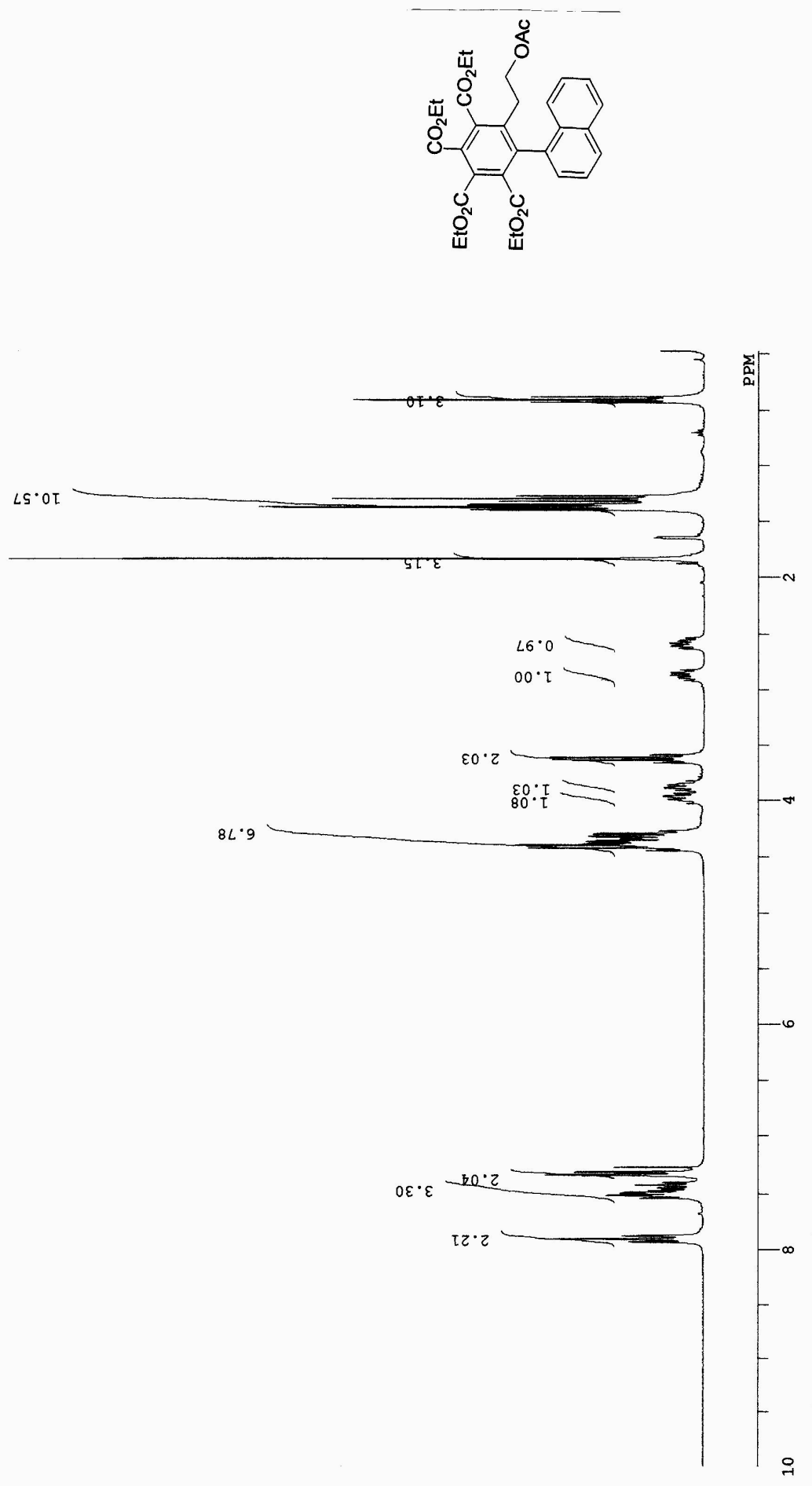